\title{
Enhanced Diversity Gain of Subcarrier Index Modulated MIMO-GFDM using Constellation Precoding Technique for $\mathbf{5 G}$
}

Relangi Anil Kumar ( $\sim$ ranilkumarphd@gmail.com )

JNTUK University

Satya Prasad K

Vignan's Foundation for Science Technology and Research

\section{Research Article}

Keywords: OMA, GFDM, SIM, Constellation Precoding, MIMO-GFDM, 5G

Posted Date: March 23rd, 2021

DOI: https://doi.org/10.21203/rs.3.rs-198564/v1

License: (c) (i) This work is licensed under a Creative Commons Attribution 4.0 International License.

Read Full License 


\title{
Enhanced Diversity Gain of Subcarrier Index Modulated MIMO- GFDM using Constellation Precoding Technique for 5G
}

\author{
${ }^{* 1}$ Relangi Anil Kumar \& ${ }^{2}$ Dr. K. Satya Prasad \\ ${ }^{{ }^{*} 1}$ Electronics \& Communication Engineering, Research scholar in JNTUK University, Assistant professor in \\ Aditya college of engineering \& technology, Surampalem. \\ ${ }^{2}$ Rector, Department of Electronics and Communication Engineering \\ Vignan's Foundation for Science, Technology \& Research (VFSTR). \\ *Email: anidecs@gmail.com
}

Abstract: The massive growth of mobile users and various applications in coming days requires an enhanced Radio Access Technology (eRAT) to improve the diversity gain and spectral efficiency. Current research on Generalized Frequency Division Multiplexing (GFDM) modulation technique combined with Multi-Input Multi-Output (MIMO) antenna system gives good resource allocation, out band emission and better signal strengths over the existing Orthogonal Multiple Access (OMA) techniques. The proposed scheme explains the improvement of diversity gain, spectral efficiency and energy efficiency of MIMO-GFMD system by combining the benefits of Subcarrier Index Modulation (SIM) and Constellation Precoding (CP) Technique. It is referred as SIM-CP-MIMO-GFDM system. In order to achieve the requirements, in proposed scheme few subcarriers are activated by index modulation bits in quadrature/in-phase dimensions and then data symbols are constellation Precoded. At the receiver, data blocks are detected by using QR Decomposition based Maximum Likelihood (ML) detection. Finally, this paper explains both theoretical and simulation result analysis that the proposed SIM-CP-MIMO-GFDM system outperforms compared to existing systems.

Keywords: OMA, GFDM, SIM, Constellation Precoding, MIMO-GFDM, 5G

\section{Introduction}

The current wireless technology applications are very popular due to exponential growth of smart phone users in the world. This is not only attracting the mobile phone users but also the developing education system, business and smart transport system all around the world [1]. Various mobile technologies such as Zero Generation (0G)-Mobile Telephone Services (MTS), First Generation (1G)-Analog cellular systems, Second Generation (2G)Digital cellular systems, Third Generation (3G) - Broadband Services and Fourth Generation (4G)- broadband with local Internet Protocol increased the user expectations due to attractive applications. But, there are some drawbacks [2] in mentioned technologies. In order to address the issues, the Fifth-Generation (5G) technologies are developed. Furthermore, it will 
become a greatest global intelligent technology also called as World Wide Wireless Web (WWWW) technology[3], [4].

The exponential growth of wireless network devices and traffic data streams motivated the scientists and research scholars to develop new technology which is focussed to satisfy the requirements of $5 \mathrm{G}$ with high spectral, energy efficiency and data rates.

This is directed to implementation of physical layer with Millimetre Wave, massive Multi-Input Multi-Output (mMIMO), Non-Orthogonal Multiple Access (NOMA) and Index Modulation (IM). Basically, the Spectral Efficiency [5] and throughput of the communication system improved using mMIMO Technology for 5G [6]. Also, NOMA is considered for future wireless communication systems which increase the spectral efficiency by allowing two users sharing same carrier [7], [8] . Also, data rates are increased due to higher bandwidth facility in Millimetre Wave technology [9].

On the other hand, IM methods are used transmission entity bits to convey the information bits for improved energy and spectral efficiency. These systems have drawbacks of reliability and coverage [10],[11]. Therefore, we are relying on a potential candidate in physical layer to improve the coverage, spectral, energy efficiency and throughput of the system. This relying reduces the path-loss and achieves the spatial diversity without deploying more number of antennas at the transmitter and receiver [12].

Orthogonal Frequency Division Multiplexing (OFDM) system is a multicarrier modulation system in which certain transmission entities are deactivated to save energy by using Index modulation. Few subcarriers are alternatively in on/off state based on index bits. On the other hand, Spatial Modulation(SM) schemes are implemented to activate/deactivate transmitting antennas based on the data bits. This concept is already employed in GFDM and OFDM systems to improve the performance gain over conventional system. These systems are referred as IM-GFDM and IM-OFDM [13], [14]. The Subcarrier Index Modulation with OFDM system reduces the Peak to Average Power Ratio (PAPR) by utilizing subcarrier indices encoded with index bits [15]. Recent studies have explained various IM development schemes based on motivation from spatial modulation techniques. They are reviewed in [16].

Next, a SM scheme has extended to Quadrature Spatial Modulation (QSM) in OFDM systems [17]. In this method, the transmitted bits are divided into real and imaginary parts for allowing simultaneous transmission over multiple antenna system to the receiver. This 
scheme improves the spectral efficiency due to the extension of spatial domain in SM. With inspiration of QSM modulation, in-phase and quadrature index modulation has been implemented with OFDM system (OFDM-IQ-IM) for allowing the ON or OFF keying approach with help of I and Q bits. Therefore, bit error rate performance and spectral efficiency is improved in OFDM-IQ-IM system [18]. Furthermore, A Generalized Precoding supported QSM (GPQSM) has been implemented in [19], in which both in-phase and quadrature phase components are allocated for the spatial modulation. Hence, the system conveyed extra index bits as compared to information bits.

Nowadays, in order to improve the both transmission diversity and spectral efficiency most of the researchers have employed multiple signal constellations for IM by considering OFDM-IM system. This multiple signal constellation approach is motivation to implement Multiple Mode (MM-OFDM-IM) scheme. In this method, to modulate the $n$ subcarriers, $n$ different sized signal constellations are used. Next, this method is extended by Wen et.al [20] for multiple signal constellations with different sizes to improve the spectral efficiency of conventional OFDM system.

These signal constellations are have been calculated from the unit circle for obtaining an optimal asymptotic BER of the Generalized (GMM-OFDM-IM) system but authors did not explain the optimal constellation size selection process for different modes of operations. Therefore, spectral efficiency enhanced only for activated subcarriers in particular mode.

The combined advantages of IM, MIMO, and OFDM transmission schemes are motivated towards the joint implementation of MIMO-OFDM-IM systems to enhance the data rates for future communication systems with favourable solution [21]. Furthermore, these systems are generalized to represent the constellation symbols and additional information bits for activate the part of subcarriers [22]. Where, stationary sub-carriers do not represent the constellation sign and also restriction of spectral efficiency in OFDM-IM methods not suitable for future communications. Hence, this article suggests the Generalized Frequency Division Multiplexing (GFDM) technique [23] due to flexible implementation in physical layer. This scheme allows to utilizing several time-slots over the subcarriers [24]. The GFDM system has advantages link reduced Out-Band-Emission (OBE) due to pulse shaping of individual subcarrier, enhanced spectral efficiency and low latency than compared to OFDM system and also it takes less operating cost to implement the block based structure 
and addition of Cyclic Prefix (CP) is possible in this scheme to avoid the Inter Symbol Interference (ISI) at the receiver [25].

Advanced research is motivated towards the GFDM system with index modulation and MIMO antenna system transmission to get the efficient data rates and spectral efficiency for $5 \mathrm{G}$ wireless networks [26]. It is further extended to improve frequency diversity. On the other hand, different coding techniques such as Space Time Coding (STC), Time ReversalSpace Time Coding (TR-STC) and Linear Precoding (LP) are combined with OFDM-IM to improve the diversity gain of the system. STC and TR-STC methods mainly transmit the signal over two independent fading channels. STC transmit the direct data sysmbols but TRSTC transmits the encoded waveform samples because of signal considered in time domain [27].

In order to improve the diversity gain without affecting the SE the Linear Precoding [28] technique is implemented along with MM-OFDM-IMIQ. This technique illustrates the more analysis needed for frequency diversity improvement in GFDM-IM system. According to literature survey of the authors, the diversity schemes for SIM-MIMO-GFDM not yet implemented anywhere. Author's expected this technique is required for high data rates transmissions with good reliability for future wireless communication systems.

Additionally, at the receiver of the OFDM/GFDM systems to detect the spatially multiplexed signals several iterative algorithms proposed [29]. Basically, Maximum Likelihood (ML) detectors, Minimum Mean Square Error (MMSE) detectors, LogLikelihood Ratio (LLR) detectors are used to achieve the optimal error performance of the received signal. Authors identified a problem; an optimal Maximum Likelihood detection scheme takes more computational complexity analysis with index modulation while increasing the time slots and users. Li et al. solved this problem in [30] by designing low complexity near-ML detection method.

Detection of MIMO-OFDM-IM signals at the receiver based on Sequential Monte Carlo (SMC) theory has been proposed in [31]. Even though, an efficient detection of ML methods is available, this leads to increased computational complexity of the system. Present research is going on linear detectors including MMSE and Linear Zero Forcing (LZF) detectors to reduce the complexity. The detection performances of MIMO-OFDM and MIMO-GFDM are very poor due to bad channel conditions and worst computational complexity $[28,[32]]$ with precoding techniques. Therefore, finally we can comprehend from 
the above discussion that GFDM system is further investigated to achieve the spectral efficiency, energy efficiency and diversity order. Hence, we proposed in this article a novel SIM-CP-MIMO-GFDM scheme that combines the advantages of MIMO transmission, subcarrier index modulation with in-phase and quadrature phase bits and constellation precoding for efficient data transmission over a multipath environment. The major contributions of this research article as summarized follows:

$\checkmark$ In this article, we proposed SIM-CP-MIMO-GFDM system. In this scheme subcarriers are activated by innovative way on both in-phase and quadrature branches to improving the spectral and energy efficiency of the system. At the same time, zero values are assigned to inactive subcarriers to save the transmission energy.

$\checkmark$ This scheme also carried out for increasing the diversity gain by constellation precoding. The diversity order is calculated from [33] minimum value of $\left(\operatorname{rank}\{K\}, \operatorname{rank}\left\{A_{\beta}\right\}\right)$. Where, $A_{\beta}=\left(x_{\beta}-\hat{x}_{\beta}\right)^{H}\left(x_{\beta}-\hat{x}_{\beta}\right)$ and $K$ is full rank covariance matrix such that $K=E\left[h_{\beta} h_{\beta}^{T}\right]$ of $h_{\beta}$. Therefore, the diversity order is decided by the $\operatorname{rank}\left\{A_{\beta}\right\}$. Suppose $x_{\beta}$ and $\hat{x}_{\beta}$ have different Subcarrier Activation Patterns (SAP) that must holds $\operatorname{rank}\left\{A_{\beta}\right\} \geq 2$. Otherwise, diversity value is limited to unity. Therefore, the paper is aiming to enhance the diversity gain by considering subcarrier activation and also applying constellation precoding on both I and Q branches simultaneously. Therefore, additionally we achieve the diversity gain by rotating constellation symbols by using constellation precoding.

$\checkmark$ The existing methods MMSE, ML and LLR detectors not suitable for proposed scheme because it spread the M-ary modulated symbols across the entire active subcarriers. Therefore, we proposed QR decomposition based ML detector for proposed method in order to get good performance in bad channel conditions and with less complexity.

The left over portions of the paper is explained as follows: The introduction of the paper is explained in section 1 and literature review is explained in section 2. The proposed SIM-CP-MIMO-GFDM system and QRD based ML detection methodology is explained in section 3. In section 4, the performance analysis of proposed system explained. Finally, conclusions are discussed in section 5 . 
Table 1: Acronyms and Abbreviations

\begin{tabular}{|c|c|}
\hline Abbreviation /Acronyms & Description \\
\hline AWGN & Additive White Gaussian Noise \\
\hline BER & Bit Error Rate \\
\hline $\mathrm{CC}$ & Channel Capacity \\
\hline $\mathrm{CP}$ & Cyclic Prefix \\
\hline $\mathrm{CP}$ & Constellation Precoding \\
\hline $\mathrm{CM}$ & Complex Multiplications \\
\hline $\mathrm{EE}$ & Energy Efficiency \\
\hline eRAT & enhanced Radio Access Technology \\
\hline GFDM & Generalized Frequency Division Multiplexing \\
\hline GPQSM & Generalized Precoding supported QSM \\
\hline ISI & Inter Symbol Interference \\
\hline LLR & Log Likelihood Ratio \\
\hline LZF & Linear zero forcing \\
\hline MM-IM & Multi-Mode Index Modulation \\
\hline mMIMO & Massive Multi Input and Multi Output \\
\hline MMSE & Minimum Mean Square Error \\
\hline ML & Maximum Likelihood \\
\hline MTS & Mobile Telephone Services \\
\hline NOMA & Non-Orthogonal Multiple Access \\
\hline OBE & Out of Band Emission \\
\hline OFDM & Orthogonal Frequency Division Multiplexing \\
\hline OMA & Orthogonal Multiple Access \\
\hline OOB & Out-Of-Band Energy \\
\hline PAPR & Peak to Average Power Ratio \\
\hline PHY & Physical Layer \\
\hline QSM & Quadrature Spatial Modulation \\
\hline SAP & Subcarrier Activation Pattern \\
\hline SC-FDE & Single Carrier Frequency Domain Equalization System \\
\hline SE & Spectral Efficiency \\
\hline
\end{tabular}




\begin{tabular}{|l|l|}
\hline SER & Symbol Error Rate \\
\hline SIC & Successive Interference Cancellation \\
\hline SIM & Subcarrier Index Modulation \\
\hline SM & Spatial Modulation \\
\hline SMC & Sequential Monte Carlo \\
\hline SRRC & Square Root Raised Cosine \\
\hline TR-STC & Time Reversal- Space Time Coding \\
\hline WWWW & World Wide Wireless Web \\
\hline
\end{tabular}

\section{Proposed Methodology}

In this section, first we will explain the generation of modulated symbols of proposed SIM-CP-MIMO-GFDM scheme at transmitter with an example. Next, these symbols are transmitted from multiple antennas. Finally, these data blocks are detected by using QR Decomposition based Maximum Likelihood (ML) detection at the receiver.

\subsection{SIM-CP-MIMO-GFDM system model}

Block diagram of proposed SIM-CP-MIMO-GFDM scheme is as shown in figure1. The mathematical analysis and functions of GFDM modulator explained in [34]. In this scheme, few subcarriers are activated by index modulation bits in quadrature/in-phase dimensions and then data symbols are constellation precoded to achieve the extra diversity order and spectral efficiency. The proposed SIM-CP-MIMO-GFDM transmitter section is as shown in figure 2.

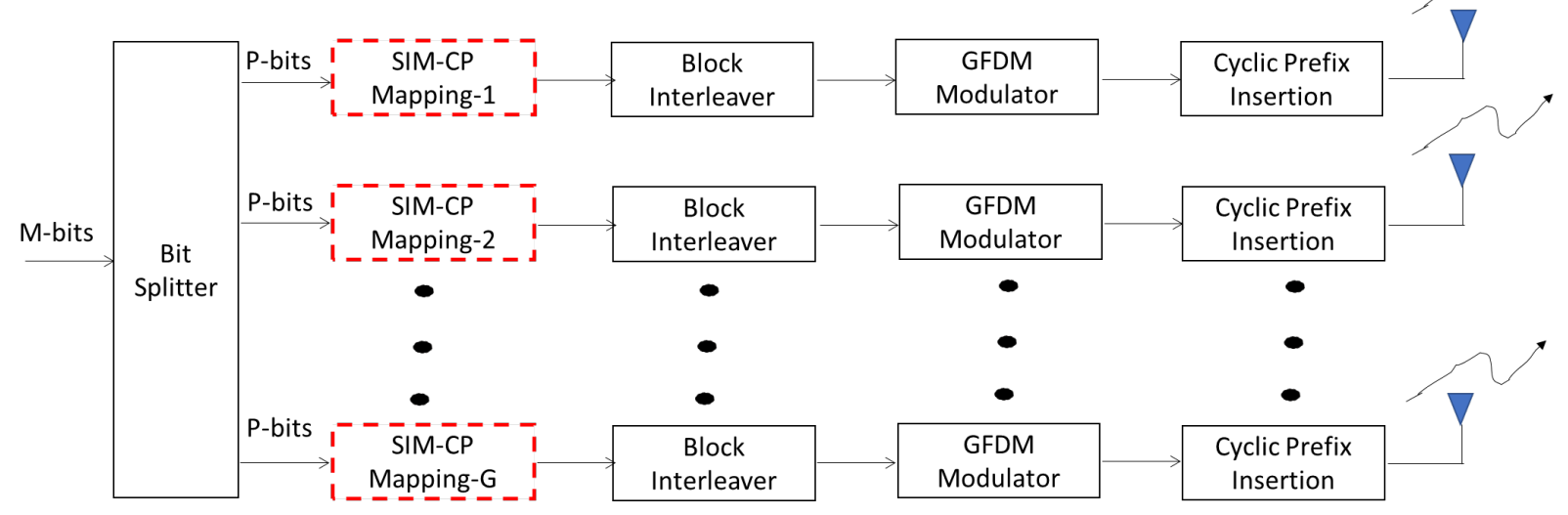

Figure 1: Proposed SIM-CP-MIMO-GFDM transmitter section 
First, the total $M$-information bits are divided into $G$ groups by using bit splitter. Each group contains $P$ number of bits such that $M=G P$. Similarly, the total GFDM sub-carriers are also divided into $G$ groups based on interleaved manner for subcarrier mapping. After SIM-CP mapping and GFDM modulation the precoded symbols are transmitted from each transmitting antenna. Operational block diagram of IM-CP mapper is as shown in figure 2.

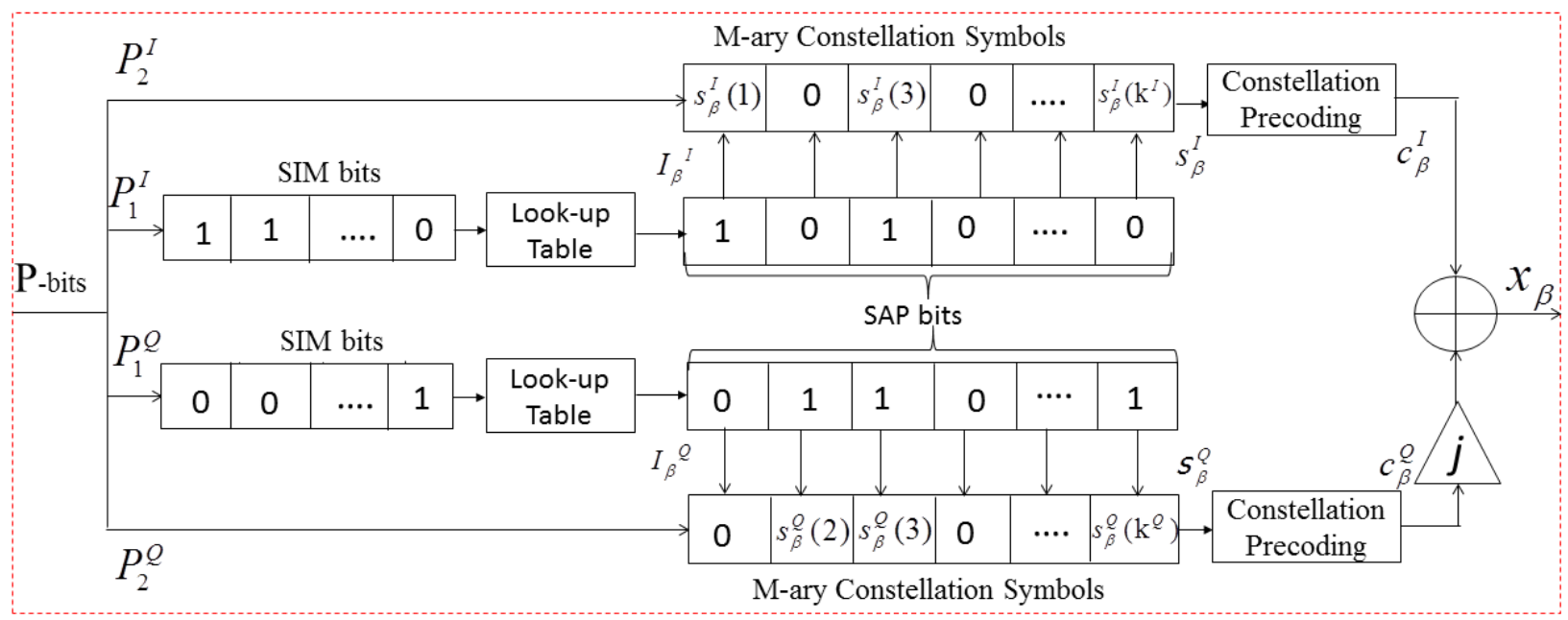

Figure 2: Block diagram of IM-CP Mapper

The total $P$ bits again sub-divided into four parts for index modulation and constellation Precoding on both in-phase and quadrature phase branches. The first section contains $P_{1}=\left\lfloor\log _{2}\left({ }^{n} C_{k^{I}}{ }^{n} C_{k^{Q}}\right)\right\rfloor$ bits. Where, represents the $\left.\rfloor\right\rfloor$ ceiling function.

When, index modulation performs on $P_{1}$ bits, in which $k^{I}$ and $k^{Q}$ active subcarriers are chosen out of $\mathrm{n}$ subcarriers to transmit the real and imaginary Subcarrier Activation Patterns (SAP's) respectively. The remaining non-selected sub-carriers are inactive during the communication. Therefore, the indices of active sub-carriers set are $I_{\beta}=\left\{I_{\beta}{ }^{I}, I_{\beta}{ }^{Q}\right\}$. Where, $I_{\beta}{ }^{I}, I_{\beta}{ }^{Q}$ represented as follows for $\beta^{\text {th }}$ group:

$$
I_{\beta}{ }^{I}=\left\{i_{\beta, 1}^{I}, \ldots . ., i_{\beta, K^{I}}^{I}\right\}, I_{\beta}^{Q}=\left\{i_{\beta, 1}^{Q}, \ldots . ., i_{\beta, K^{Q}}^{Q}\right\} .
$$

From figure 2, the remaining two branches contain $P_{2}^{I}=k^{I} \log _{2}(M)$ and $P_{2}^{Q}=k^{Q} \log _{2}(M)$ bits. Where, $\mathrm{M}$ is digital modulation order. When, bits are passed through $\mathrm{M}$-ary constellation mapper it is generate the $k^{I}$ and $k^{Q}$ real and imaginary symbol signs respectively for $\beta^{\text {th }}$ group as shown in below equation (2). 


$$
s_{\beta}^{I}=\left[s_{\beta}^{I}(1), \ldots, s_{\beta}^{I}\left(k^{I}\right)\right]^{T} ; s_{\beta}^{Q}=\left[s_{\beta}^{Q}(1), \ldots, s_{\beta}^{Q}\left(k^{Q}\right)\right]^{T}
$$

The In-phase and quadrature phase subcarrier activation patterns are added together to form a composite signal. First, this article carried out the mathematical analysis of the proposed scheme without using constellation precoding technique. The equation $x_{\beta}(\lambda)=x_{\beta}^{I}(\lambda)+j x_{\beta}^{Q}(\lambda)$ is a created block in frequency domain representation for $\beta^{\text {th }}$ group. Then the block $x_{\beta}(\lambda)$ is rearranged by a matrix Interleaver block such that the matrix multiplication is possible with GFDM modulation matrix. These elements are fed into row matrix with order $1 X N$ then multiplied by $N X N$ GFDM modulation matrix. After modulation, the cyclic prefix is inserted to every GFDM block in order to avoid the Inter Symbol Interference (ISI) at the receiver and then up-converted signals are transmitted over the multipath environment.

The received signal is as modelled in equation (3) for $\alpha^{\text {th }}$ subcarrier in the presence of Additive White Gaussian Noise (AWGN) after removing the cyclic prefix.

$$
y(\alpha)=h(\alpha) x(\alpha)+w(\alpha), \quad \alpha=1,2, \ldots ., N .
$$

Where, $w(\alpha)$ and $h(\alpha)$ are complex noise sample and channel frequency response at $\alpha^{\text {th }}$ subcarrier respectively. Equation (3) is solved by using optimal ML detector which is independently decode the $\hat{I}_{\beta}, \hat{s}_{\beta}^{I}, \hat{s}_{\beta}^{Q}$ symbols for $\beta^{\text {th }}$ group. Finally, at end of the receiver $\hat{I}_{\beta}$ is demapped as index modulation bits and $\left\{\hat{s}_{\beta}^{I}, \hat{s}_{\beta}^{Q}\right\}$ are demodulated as an ordinary constellation sysmbols. It is concluded, that the equation (4) takes exhaustive search for I and Q- Subcarrier Activation Patterns (SAP) and needed more complex multiplications. For better performance, the low complexity detectors explained in section 3 .

$$
\begin{aligned}
& \left(\hat{I}_{\beta}, \hat{s}_{\beta}^{I}, \hat{s}_{\beta}^{Q}\right)=\underset{I_{\beta}, s_{\beta}^{I}, s_{\beta}^{O}}{\arg \min } \sum_{k=1}^{k^{I}}\left|h\left(i_{\beta, \mathrm{k}}^{I}\right)\right|^{2}\left[s_{\beta}^{I}(\mathrm{k})\left(s_{\beta}^{I}(\mathrm{k})-2 r^{I}\left(i_{\beta, \mathrm{k}}^{I}\right)\right)\right]+\sum_{v=1}^{k^{Q}}\left|h\left(i_{\beta, v}^{Q}\right)\right|^{2}\left[s_{\beta}^{Q}(v)\left(s_{\beta}^{Q}(v)-2 r^{Q}\left(i_{\beta, v}^{Q}\right)\right)\right] \\
& \text { Where, } r(\alpha) \square r^{I}(\alpha)+j r^{Q}(\alpha)=\frac{\mathrm{y}(\alpha)}{h(\alpha)} \quad \alpha=1,2 \ldots \mathrm{N} \text { subcarriers } \\
& r_{\beta}(\lambda)=r(\beta+(\lambda-1) g), \quad \beta=1,2, \ldots, g \text { groups and } \lambda=1,2 \ldots \mathrm{n} \text { active subcarriers }
\end{aligned}
$$

\subsubsection{Spectral Efficiency}

According to literature review, most of the cases an index bits are used for activate the antenna indices in MIMO systems. Which is provides the maximum first and second order 
diversity order only [33]. These are not suitable for spatial modulation systems. On other hand, the researchers and scientist are concentrated to use index bits for activate the subcarriers in present technology. The spectral efficiency of GFDM-IM system is calculated as shown in equation (5) measured in bits per second per $\mathrm{Hz}$.

$$
\text { Spectral Efficiency }(S E)=\frac{p_{1}+p_{2}}{n}=\frac{\left\lfloor\log _{2}\left({ }^{n} C_{k}\right)\right\rfloor}{n}+\frac{k}{n} \log _{2}(M) .
$$

Here, first term present the bits related to IM and second term represent the bits related to M-ary modulation. Due to existence of exponent $\mathrm{k}$ in second term $\left(M^{k}\right)$, this grows faster than the first term $\log \left({ }^{n} C_{k}\right)$ and both terms limited the spectral and energy efficiency of GFDM-IM system. In order to increase the spectral efficiency of the proposed technique along with constellation precoding the subcarriers are activated by innovative way on both In-phase (I) and Quadrature (Q) branches. Therefore, the extra terms $\frac{\log _{2}{ }^{n} C_{k^{Q}}}{n}+\frac{k^{Q}}{n} \log _{2}(M)$ in equation (6) improving the spectral efficiency of our proposed scheme as compared to existing system.

$$
\text { Spectral Efficiency }=\frac{p_{2}^{I}+p_{2}^{Q}+p_{1}}{n}=\frac{\left\lfloor\log _{2}\left({ }^{n} C_{k^{Q}} \cdot{ }^{n} C_{k^{Q}}\right)\right\rfloor}{n}+\frac{k^{I}+k^{Q}}{n} \log _{2}(M)
$$

\subsubsection{Diversity Gain}

Related to previous work, there are few drawbacks in GFDM with index modulation. After transmitting the symbols, every sub-carrier within a group experienced the independent channel effect. Hence defined two diagonal matrices $x_{\beta}$ and $\hat{x}_{\beta}$ with order $n x n$ for $\beta^{\text {th }}$ group in frequency domain is given as $\left[x_{\beta}(1), x_{\beta}(2), \ldots, x_{\beta}(\mathrm{n})\right]^{T}$ and $\left[\hat{x}_{\beta}(1), \hat{x}_{\beta}(2), \ldots, \hat{x}_{\beta}(\mathrm{n})\right]^{T}$ respectively. Where, $x_{\beta}(\lambda)=x(\beta+(\lambda-1) \mathrm{g})$ and $\hat{x}_{\beta}(\lambda)=\hat{x}(\beta+(\lambda-1) \mathrm{g})$ Where, $\lambda=1,2, \ldots n$. The diversity order is calculated from [33] minimum value of $\left(\operatorname{rank}\{K\}, \operatorname{rank}\left\{A_{\beta}\right\}\right)$ for existing OFDM-IM, GFDM-IM and GFDM-IQIM systems. Where, $A_{\beta}=\left(x_{\beta}-\hat{x}_{\beta}\right)^{H}\left(x_{\beta}-\hat{x}_{\beta}\right)$ and $K$ is full rank covariance matrix such that $K=E\left[h_{\beta} h_{\beta}^{T}\right]$ of $h_{\beta}$.

Therefore, the diversity order is decided by the $\operatorname{rank}\left\{A_{\beta}\right\}$. Suppose $x_{\beta}$ and $\hat{x}_{\beta}$ have different SAP's that holds $\operatorname{rank}\left\{A_{\beta}\right\} \geq 2$. Otherwise, diversity value is limited to unity. 
Therefore, the paper is aiming to enhance the diversity gain by applying Constellation Precoding (CP) to the M-ary Modulated symbols explained in section (2.2).

\subsection{Constellation Precoded SIM-MIMO-GFDM}

In order to increase the order of the diversity gain, in this section an optimal precoding technique is explained along with Subcarrier Index Modulation. Hence proposed scheme is referred as SIM-CP-MIMO-GFDM. From equation (2) $s_{\beta}^{I}$ and $s_{\beta}^{Q}$ are M-ary modulated symbols which are input to the Constellation Precoder. This is performed on each I and Q-branches. A constellation precoder is as shown in equation (), where elements belong to $\Re$ and $b_{i 1}^{2}+b_{i 2}^{2}=1, i=1,2$ is constrain, condition to satisfy the power same as input.

$$
\begin{gathered}
B=\left[\begin{array}{ll}
b_{11} & b_{12} \\
b_{21} & b_{22}
\end{array}\right] \quad b_{i 1}^{2}+b_{i 2}^{2}=1, i=1,2 \\
\text { Where, } b_{11}^{*}= \pm \operatorname{cosp}(M), b_{12}^{*}= \pm \operatorname{sinp}(M), b_{21}^{*}= \pm \operatorname{sinp}(M), b_{22}^{*}= \pm \operatorname{cosp}(M) \\
\text { Where, } p(M)=\frac{1}{2} \arcsin \left(\frac{2(M+1)\left(M^{2}-2 M+2\right)-4(M-2) \sqrt{M^{3}}}{(M-1)^{4}+2(M-1)^{2}+16(M-1)+17}\right)
\end{gathered}
$$

Equation (9) represent the optimization of precoding angle for modulation order $M$. Instead of directly transmitting M-ary modulation symbols over In-phase and quadrature branches of a subcarrier, in this method a pair of symbols are generated using equations (7) and then transmitted over I/Q branch of active subcarrier. Where, diamond shaped or rotated M-ary constellation symbols are calculated with precoding technique. Therefore, diversity order is doubled. The mathematical analysis carried out only on in-phase branch because it is same as quadrature branch. The $s_{\beta}^{I}=\left[s_{\beta}^{I}(1), \ldots, s_{\beta}^{I}\left(k^{I}\right)\right]^{T}$ symbols are generated by passing $p_{2}^{I}$ bits through M-ary modulator of $\beta^{\text {th }}$ group. After that $s_{\beta}^{I}$ is divided into pairwise and then multiplication is carried out by constellation precoding matrix as shown in equation (10) 


$$
c_{\beta}^{I}=\left[\begin{array}{c}
c_{\beta}^{I}(1) \\
c_{\beta}^{I}(2) \\
\vdots \\
c_{\beta}^{I}\left(k^{I}-1\right) \\
c_{\beta}^{I}\left(k^{I}\right)
\end{array}\right]=\left[\begin{array}{c}
B\left[\begin{array}{c}
s_{\beta}^{I}(1) \\
s_{\beta}^{I}(2)
\end{array}\right] \\
\vdots \\
B\left[\begin{array}{c}
s_{\beta}^{I}\left(k^{I}-1\right) \\
s_{\beta}^{I}\left(k^{I}\right)
\end{array}\right]
\end{array}\right]=\left[\begin{array}{c}
b_{11} s_{\beta}^{I}(1)+b_{12} s_{\beta}^{I}(2) \\
b_{21} s_{\beta}^{I}(1)+b_{22} s_{\beta}^{I}(2) \\
\vdots \\
b_{11} s_{\beta}^{I}\left(k^{I}-1\right)+b_{12} s_{\beta}^{I}\left(k^{I}\right) \\
b_{21} s_{\beta}^{I}\left(k^{I}-1\right)+b_{22} s_{\beta}^{I}\left(k^{I}\right)
\end{array}\right] .
$$

Based on successive activation pattern of index $I_{\beta}^{I}$ bits, $c_{\beta}^{I}$ information symbols are transmitted over $k^{I}$ active subcarriers with help of equation $c_{\beta}^{I}(k)=x^{I}\left(i_{\beta, k}^{I}\right), k=1, \ldots, k^{I}$. For a given $I_{\beta}^{I}$, the elements of $s_{\beta}^{I}$ are independent to each other. At the receiver, the frequency domain received signal in equation (11) is represented for $\alpha^{\text {th }}$ subcarrier.

$$
r(\alpha)=h(\alpha) x(\alpha)+w(\alpha), \quad \alpha=1,2, \ldots, \mathrm{N}
$$

Where, $h(\alpha)$ and $w(\alpha)$ represents frequency response of channel and AWGN noise samples of $\alpha^{\text {th }}$ subcarrier. After substituting $x(\alpha)$ and $h(\alpha)$ for $\beta^{\text {th }}$ group, in-phase detected components $\left(\widehat{I}_{\beta}^{I}, \widehat{s}_{\beta}^{I}\right)$ are obtained using optimal ML detection as represented in equations (12) and (13).

$$
\begin{aligned}
& \left(\widehat{I}_{\beta}^{I}, \widehat{s}_{\beta}^{I}\right)=\underset{I_{\beta}^{I}, s_{\beta}^{I}}{\arg \min } \sum_{\lambda=1}^{n}\left|h_{\beta}(\lambda)\right|^{2}\left[r_{\beta}^{I}(\lambda)-x_{\beta}^{I}(\lambda)\right]^{2}=\underset{I_{\beta}^{I}, s_{\beta}^{I}}{\arg \min } \sum_{k=1}^{k^{I}}\left|h\left(i_{\beta, k}^{I}\right)\right|^{2} c_{\beta}^{I}(k)\left[c_{\beta}^{I}(k)-2 r^{I}\left(i_{\beta, k}^{I}\right)\right] . \\
& \left(\widehat{I}_{\beta}^{I}, \widehat{s}_{\beta}^{I}\right)=\underset{I_{\beta}^{I}, s_{\beta}^{I}}{\arg \min } \sum_{\xi=1}^{\frac{k_{I}}{2}}\left|h\left(i_{\beta, 2 \xi-1}^{I}\right)\right|^{2} c_{\beta}^{I}(2 \xi-1)\left[c_{\beta}^{I}(2 \xi-1)-2 r^{I}\left(i_{\beta, 2 \xi-1}\right)\right]+\left|h\left(i_{\beta, 2 \xi}^{I}\right)\right|^{2} c_{\beta}^{I}(2 \xi)\left[c_{\beta}^{I}(2 \xi)-2 r^{I}\left(i_{\beta, 2 \xi}\right)\right]
\end{aligned}
$$

Here, $r_{\beta}^{I}(\lambda)=r(\beta+(\lambda-1) G), \quad \beta=1,2, \ldots \mathrm{G}, \lambda=1,2, \ldots \mathrm{n}$.

$\operatorname{alsoc} c_{\beta}^{I}(2 \xi-1)=b_{11} s_{\beta}^{I}(2 \xi-1)+b_{12} s_{\beta}^{I}(2 \xi)$ and $c_{\beta}^{I}(2 \xi)=b_{21}(2 \xi-1)+b_{22} s_{\beta}^{I}(2 \xi)$

Where, $2 \xi-1,2 \xi$ are odd and even index terms respectively. Pairwise symbols are generated by $\xi=1,2, \ldots . k^{I} / 2$ As a result the computational complexity of the ML detected equation is in the order of $O\left(C\left(n, k^{I}\right) M^{2} k^{I} / 2 n\right)$ per subcarrier and this increases for larger values of $\mathrm{n}$ and $k^{I}$.In order to significantly reduce the computational complexity, the recent research 
suggested Log Likelihood Ratio (LLR) detector. After employing this method on equation (11), within a group all subcarriers calculated as follows:

$$
L_{\beta}^{I}(\lambda) \alpha \ln \left(\sum_{u \in U_{1} \cup U_{2}} e^{-\frac{\left|h_{\beta}(\lambda)\right| H 2}{N_{0}}\left(r_{\beta}^{I}(\lambda)-u\right)^{2}}\right)+\frac{\left|h_{\beta}(\lambda) r_{\beta}^{I}(\lambda)\right|^{2}}{N_{0}}, \lambda=1,2, \ldots, n .
$$

Where, $U_{I}=\left\{b_{11} s_{1}+b_{12} s_{2}, \forall s_{1}, s_{2} \in Z\right.$ and $U_{2}=\left\{b_{21} s_{1}+b_{22} s_{2}, \forall s_{1}, s_{2} \in Z\right\}$ are formed to display this. The modulated symbols are estimated pair by pair by using following expression (15)

$$
\left(\widehat{s}_{\beta}^{I}(2 \xi-1), \widehat{s}_{\beta}^{I}(2 \xi)\right)=\underset{s_{\beta}^{I}(2 \xi-1), s_{\beta}^{I}(2 \xi)}{\arg \min }\left|h\left(\widehat{i}_{\beta, 2 \xi-1}^{I}\right)\right|^{2}\left[r^{I}\left(\widehat{i}_{\beta, 2 \xi-1}^{I}\right)-c_{\beta}^{I}(2 \xi-1)\right]^{2}+\left|h\left(\widehat{i}_{\beta, 2 \xi}^{I}\right)\right|^{2}\left[r^{I}\left(\widehat{i}_{\beta, 2 \xi}^{I}\right)-c_{\beta}^{I}(2 \xi)\right]^{2}
$$

Where, $\xi=1,2, \ldots, k^{I} / 2$. Equations (14) and (15) are involve in $2 n M^{2}$ and $M^{2} k^{I} / 2$ seeks.

\section{QR Decomposition based ML detection}

In this section, QR decomposition based detection method is extended to $\mathrm{ML}$ detection to reduce the computational complexity and achieve the better performance of the system. The SIM-LP-MIMO-GFDM receiver diagram is as shown in Figure 4. After removing Cyclic Prefix, the wireless channel stays stable with a single SIM-LP-MIMOGFDM receiver block and the given below equation (16) expresses the whole received signal:

$$
y(\alpha)=h(\alpha) x(\alpha)+w(\alpha), \quad \alpha=1,2, \ldots, N .
$$

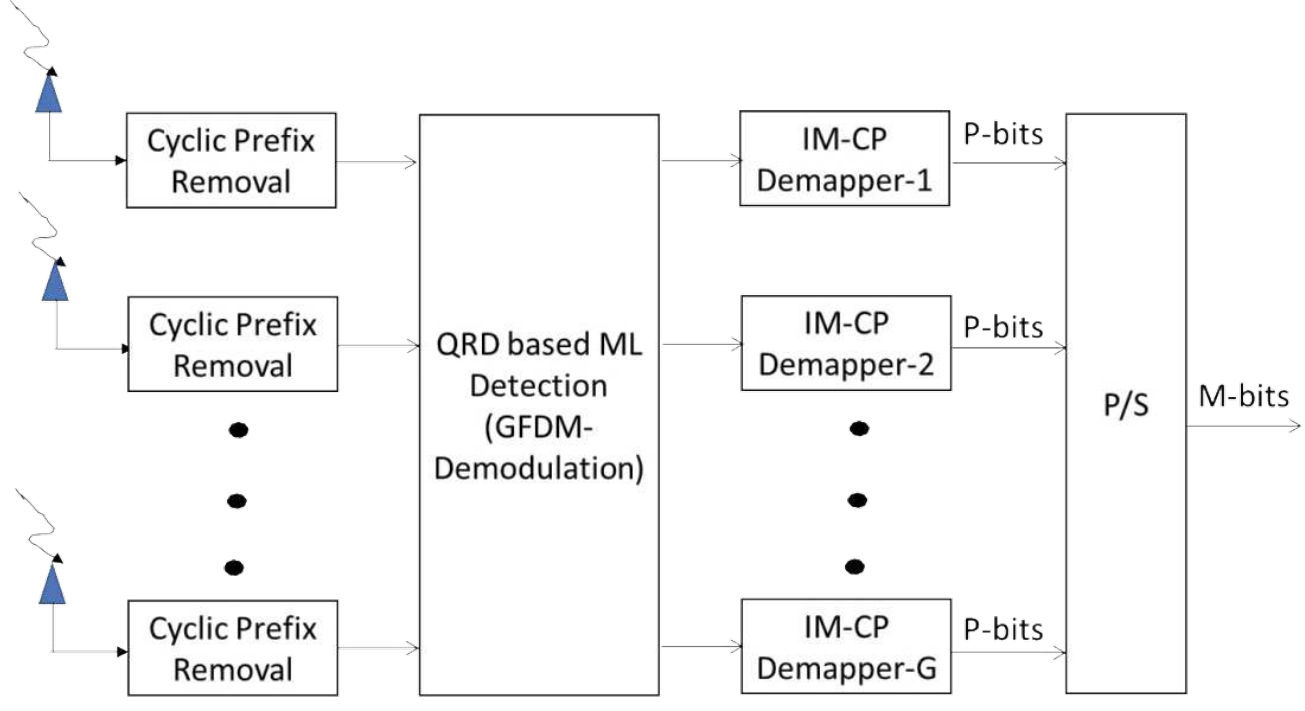

Figure 5: SIM-LP-MIMO-GFDM receiver with QR decomposition based ML detection 
Where, $w(\alpha)$ and $h(\alpha)$ represent the Additive White Gaussian Noise (AWGN) and Channel Frequency Response (CFR) for subcarrier $\alpha$. Also, $C N(0,1)$ and $C N\left(0, N_{0}\right)$ are assumed as distribution of $h(\alpha)$ and $w(\alpha)$ respectively and $N_{0}$ represent the noise variance. Using optimal ML detector for $\beta^{\text {th }}$ group obtained as following expression

$$
\begin{array}{r}
\left(\hat{I}_{\beta}, \hat{s}_{\beta}\right)=\underset{I_{\beta}, s_{\beta}}{\arg \min } \sum_{\lambda=1}^{n}\left|y_{\beta}(\lambda)\right|^{2}+\sum_{\gamma=1}^{k}\left|h\left(i_{\beta, \gamma}\right) s_{\beta}(\gamma)\right|^{2}-2 \sum_{\gamma=1}^{k} \mathfrak{R}\left\{y^{*}\left(i_{\beta, \gamma}\right) h\left(i_{\beta, \gamma}\right) s_{\beta}(\gamma)\right\}, \\
\text { Where, } y_{\beta}(\lambda)=y(\beta+(\lambda-1) g), \beta=1,2, \ldots ., g, \lambda=1,2, \ldots, n
\end{array}
$$

In general, the estimation of equation (17) takes the complexity order $O\left(C(n, k) M^{k} / n\right)$ per subcarrier. This is increased exponential with $\mathrm{M}$ and increased linearly with $C(n, k)$. Hence, the major concern of QR decomposition method is suggested to detect the $\hat{I}_{\beta}, \hat{s}_{\beta}^{I}, \hat{s}_{\beta}^{Q}$ GFDM blocks. The SIM-CP-MIMO-GFDM data blocks are received by multiple receiving and then after QR decomposition based ML scheme the original information bits are detected by IM-CP de-mappers at end of the receiver. The proposed scheme achieved good detection performance with higher signal to noise ratio values.

QR decomposition based ML detection algorithm as explained follows. The channel matrix is extended using QR decomposition [35] as shown below equation (18).

$$
H=\left[\begin{array}{l}
\tilde{H} \Pi \\
\sigma_{n} I_{N T}
\end{array}\right]=Q R=\left[\begin{array}{l}
Q_{1} \\
Q_{2}
\end{array}\right] R=\left[\begin{array}{l}
Q_{1} R \\
Q_{2} R
\end{array}\right]
$$

Where, $Q$ is $\operatorname{an}(N T+N R) \times N T$ order unitary matrix. This is further portioned into $Q_{1}$ and $Q_{2}$ matrix with order $N R \times N T$ and $N T \times N T$ respectively. For the sake of identifying the interleaving $\Pi$ permutes columns of before $\hat{H}$ to decomposition. From the above equation the relation holds $R=Q^{H} . H$.

$$
\begin{aligned}
& R=\left[\begin{array}{ll}
Q_{1}{ }^{H} & Q_{2}{ }^{H}
\end{array}\right]\left[\begin{array}{c}
\tilde{H} \Pi \\
\sigma_{n} I_{N T}
\end{array}\right] \\
& \therefore R=Q_{1}{ }^{H} \cdot H \Pi+Q_{2}{ }^{H} \sigma_{n}
\end{aligned}
$$


In order to detect the original information sysmbols the $Q_{1}^{H}$ matrix is multiplied with received signal as shown in below equation (21)

$$
\tilde{y}=Q_{1}^{H} y=Q_{1}^{H}(H \hat{d}+w)=Q_{1}^{H} H \hat{d}+Q_{1}^{H} w
$$

From equation (20), $\tilde{y}_{\text {can }}$ be rewritten as

$$
\tilde{y}=R \hat{d}-\underbrace{\sigma_{n} Q_{2}^{H} d+Q_{1}^{H} w}_{\text {Interference terms }} .
$$

Where, first term is useful term and remaining terms are belongs to interferences. And also equation (18) hold the relation $R^{-1}=Q_{2} / \sigma_{n}$. Where, $Q_{2}$ is upper triangle matrix useful to suppress the interferences and maximize the signal power for each layer in optimum Successive Interference Cancelation (SIC) methods. Therefore, we achieved minimum mean square errors for each layer in detection step with help of error covariance matrix $\Phi$.

$$
\Phi=\sigma_{n}^{2} \cdot\left(\mathrm{H}^{H} \mathrm{H}\right)^{-1}=\sigma_{n}^{2} \cdot \mathrm{R}^{-1} R^{-H}
$$

After minimization of interference in equation, the useful signal approximately we can write as follows:

$$
\tilde{y} \approx R \hat{d}+\tilde{w}, \quad \text { where, } \tilde{w}=Q_{1}^{H} w
$$

Using configuration of upper triangle matrix $R$, the SIM-GFDM block is used to detect the symbol $\tilde{d}_{t}^{l}$ which is corresponding to $t^{\text {th }}$ transmit antenna for $l^{\text {th }}$ sub-block of SIMCP-MIMO-GFDM scheme by using following equation (25). Where, notation $\|.\|^{2}$ is norm of the detected symbol.

$$
\widetilde{d}_{t}^{l}=\underset{d_{t}^{l} \in\{0, S\}}{\arg \min }\left\|\widetilde{y}_{t}^{l}-R_{t}^{l} d_{t}^{l}\right\|^{2} .
$$

Here, $\tilde{y}_{t}^{l}, R_{t}^{l}$ and $d_{t}^{l}$ are $n \times 1, n \times n$ and $n \times 1$ vectors respectively. The term $d_{t}^{l}$ is obtained from final columns and $n$ rows of matrix $R$. 


$$
\tilde{y}_{t}^{l}=R_{:, u} \tilde{d}_{t}^{l}
$$

Where, $R_{t}^{l}$ is an upper triangular matrix with dimensions $n \times n$. In order to takes the final $n$ and every element (row/columns) of the matrix with help of subscripts $u$ and :, notations.

$$
\tilde{y} \leftarrow \tilde{y}-\hat{y}_{t}^{l}
$$

Because of row/column wise operations the size of the system is reduced by $n$ as shown in below expression.

$$
\tilde{y} \leftarrow \tilde{y}_{\widetilde{n}}, R \leftarrow R_{\widetilde{n}, \tilde{n}} .
$$

This detection process continues for all the subsequent SIM-GFDM sub blocks until all the blocks are completed successfully. Where $\tilde{n}$ is a last $\mathrm{n}$ element of sub-scripted object.

\subsection{Computational Complexity analysis}

In this section, the proposed QR decomposition based near maximum likelihood detection computational complexity performance is compared with MMSE-LLR detector, MMSE detector and ML detector based number of multiplications. The following stages are describes the complexity of proposed detection scheme for SIM-CP-MIMO-GFDM system.

\section{Matrix $\widetilde{H}$ formulation stage:}

In proposed method, the MIMO-GFDM system contains $T$ number of transmit antennas and $R$ number of receive antennas then the channel impulse response among receiver and transmitter is represented with circular convolution matrix $\tilde{H}$ with dimension $N R \times N T$. Every row vector in $\widetilde{H}$ contains non-zero elements with length $N_{\text {delay }}$. Where, $N_{\text {delay }}$ is length of the channel delay spread. In order to calculate computational complexity let $\zeta_{i \times j}$ and $\varphi_{i \times j}$ are matrices with dimensions $i \times j$. Next, $\zeta_{i \times 1}$ and $\varphi_{i \times 1}$ are selected $i \times 1$ row vectors from $\zeta_{i \times j}$ and $\varphi_{i \times j}$ matrices. Therefore, $\widetilde{H}$ formulation stage is calculated by multiplying two matrices $\varphi_{N \times N} \times \zeta_{N \times N}$. Also, considering number of executions required for this process, $N_{\text {delay }} N^{2} T R$ number of complex multiplications needed to formulate the $\widetilde{H}$. 


\section{MMSE-QR decomposition stage:}

MMSE detection is combined with QR decomposition for better performance of the SIM-CP-MIMO-GFDM system. This stage performs the $Q R\left(\varphi_{(N T+N R) \times N T}\right)$ operation. Generally, $2 i j^{2}$ number of multiplications required to calculate $Q R$-decomposition if order of the matrix is $i \times j$. Therefore, the complexity of MMSE-QR decomposition stage represented as $2 N^{3} T^{2}(T+R)$

\section{The received signal vector modification stage:}

The dimension of $Q$ matrix is $(N T+N R) \times N T$. At the receiver, we need to compute $\varphi_{N R \times 1} \varphi_{N R \times N T}^{H}$ operations to perform the detection. Therefore, complexity of received signal vector modification is $N^{2} R T$

\section{ML detection stage:}

In this stage, Maximum Likelihood detection requires $\min \left(\| \varphi_{n \times 1}-\left.\left(\varphi_{n \times n} \zeta_{n \times 1}\right)\right|^{2}\right)$ computations. The number of multiplications needed for this process is $(N / n) c M^{\left(k^{I}+k^{Q}\right)} T$. Where, $\mathrm{c}$ is $2^{P_{1}}$ possible combinations in an index modulation but it take only $C(n, k)$ combinations. On other hand, $P_{2}^{I}$ and $P_{2}^{Q}$ bits takes $k^{I}$ and $k^{Q}$ constellation symbols respectively. Therefore, the complexity of this , Maximum Likelihood detection stage is epitomized as $(N / n)\left(c M^{\left(k^{I}+k^{Q}\right)} T\left(n\left(k^{I}+k^{Q}\right)+n\right)\right)$.

\section{SIC detection stage:}

Moreover, this stage performs the $\phi_{N T \times 1}-\phi_{N T \times n} \zeta_{n \times 1}$ subtraction to cancel the interference in the received signal. The row vector $\zeta$ contains only $k$ number of non-zero elements. The execution count of this process is computed as $(N / n) T$. Hence, the complexity of SIC detection stage is computed as $N^{2} T^{2} n /\left(k^{I}+k^{Q}\right)$. Finally, by combining the complexity of the all stages, the overall complexity of proposed detection scheme is evaluated as $O\left(N^{3}\left(2 T^{3}+2 T^{2} R\right)\right)$.

Table 2 represent the comparison of computational complexity among different detectors. From the table, it is observed that the ML-detector takes more number of complex 
multiplications but MMSE and QR decomposition based ML-detector approximately takes same number of multiplications. GFDM is a block based multicarrier modulation scheme. Therefore, at the receiver side QR-decomposition based ML detector is used to detect the GFDM blocks with minimum errors as compared to existing methods.

Table 2 Summary of the complaxity of different detector in tems of complex multiplications

\begin{tabular}{|l|l|}
\hline Type of the Detector & Number of Multiplications \\
\hline QR decomposition based ML-detector & $\sim O\left(N^{3}\left(2 T^{3}+2 T^{2} R\right)\right)$ \\
\hline MMSE detector & $\sim O\left(N^{3}\left(T^{3}+2 T^{2} R\right)\right)$ \\
\hline ML detector & $\left(c M^{\left(k^{I}+k^{Q}\right)}\right)^{T N / n}\left(\left(N^{2} R T\left(\left(k^{I}+k^{Q}\right) / n\right)+N T\right)\right.$ \\
\hline
\end{tabular}

\section{Performance Analysis of SIM-CP-MIMO-GFDM}

In this section, the performance and effectiveness of proposed SIM-LP-MIMOGFDM system is explained by comparing with existing OFDM, GFDM, OFDM-IM, GFDMIM systems by taking results on MATLB working platform. We are evaluated few performance matrices of the system such as spectral efficiency, energy efficiency, channel capacity and finally bit error rate for different detectors by assuming channel is Rayleigh fading and frequency selective. To obtain the validated results, assumed simulation parameters listed in Table 2.

Table 2: Simulation Parameters

\begin{tabular}{|l|l|}
\hline \multicolumn{1}{|c|}{ Parameters } & \multicolumn{1}{c|}{ Values } \\
\hline Input bits & 1024 \\
\hline M-ary Constellation & QAM \\
\hline Order of Precoding Matrix & $2 \mathrm{X} 2$ \\
\hline Number of subcarriers & 256 \\
\hline Transmitting antennas & 4 \\
\hline Receiving antennas & 4 \\
\hline Channel model & Rayleigh fading channel \\
\hline
\end{tabular}




\begin{tabular}{|l|l|}
\hline Bandwidth & $20 \mathrm{MHz}$ \\
\hline Cyclic prefix & $7 \%$ of data \\
\hline Modulation & OFDM and GFDM \\
\hline
\end{tabular}

\section{Spectral Efficiency}

For given a communication system, spectral efficiency is defined as the maximum throughput per unit resource spectrum. Here, throguput depends on type of the modulation, bandwidth and MIMO antennas system. This paprameter computes the how the data bits are restrcted to frequency spectrum in physical layer. The spectral efficiency of proposed SIMCP-MIMO-GFDM scheme comuted as follows:

Spectral Efficiency $=\frac{C}{B}=\frac{p_{2}^{I}+p_{2}^{Q}+p_{1}}{n}=\frac{\left\lfloor\log _{2}\left({ }^{n} C_{k^{Q}} \cdot{ }^{n} C_{k^{Q}}\right)\right\rfloor}{n}+\frac{k^{I}+k^{Q}}{n} \log _{2}(M)$

The first term is calculated from Index Modulation bits and second term is from Mary modulation symbols. Where, $\mathrm{C}$ and $\mathrm{B}$ are represent the Channel Capacity and Bandwidth respectively. In proposed method, subcarrier activation patterns are transmiited on both Inphase (I) and Quadrature (Q) branches. Therefore, the extra term in above equation () $\frac{\log _{2}{ }^{n} C_{k^{Q}}}{n}+\frac{k^{Q}}{n} \log _{2}(M)$ is increasing the spectral efficiency as compared to existing system.

Figure 4 represent the comparison analysis of spectral efficiency with different systems over active number of subcarriers. GFDM use single cyclic prefix for the entire symbol block containing $\mathrm{k}$ subcarriers to avoid the ISI at the receiver. Hence, we achieve the more SE as compared to OFDM. Also, by employing index modulation and constellation precoding on GFDM system there will be an extra benefit on spectral efficiency. Hence, SE of SIM-CP-MIMO-GFDM method has been improved up to $20.8 \mathrm{bps} / \mathrm{Hz}$. Therefore we concluded that the proposed scheme as shown in figure 4 outperforms compared to existing method like OFDM, OFDM-IM, GFDM and GFDM-IM. 


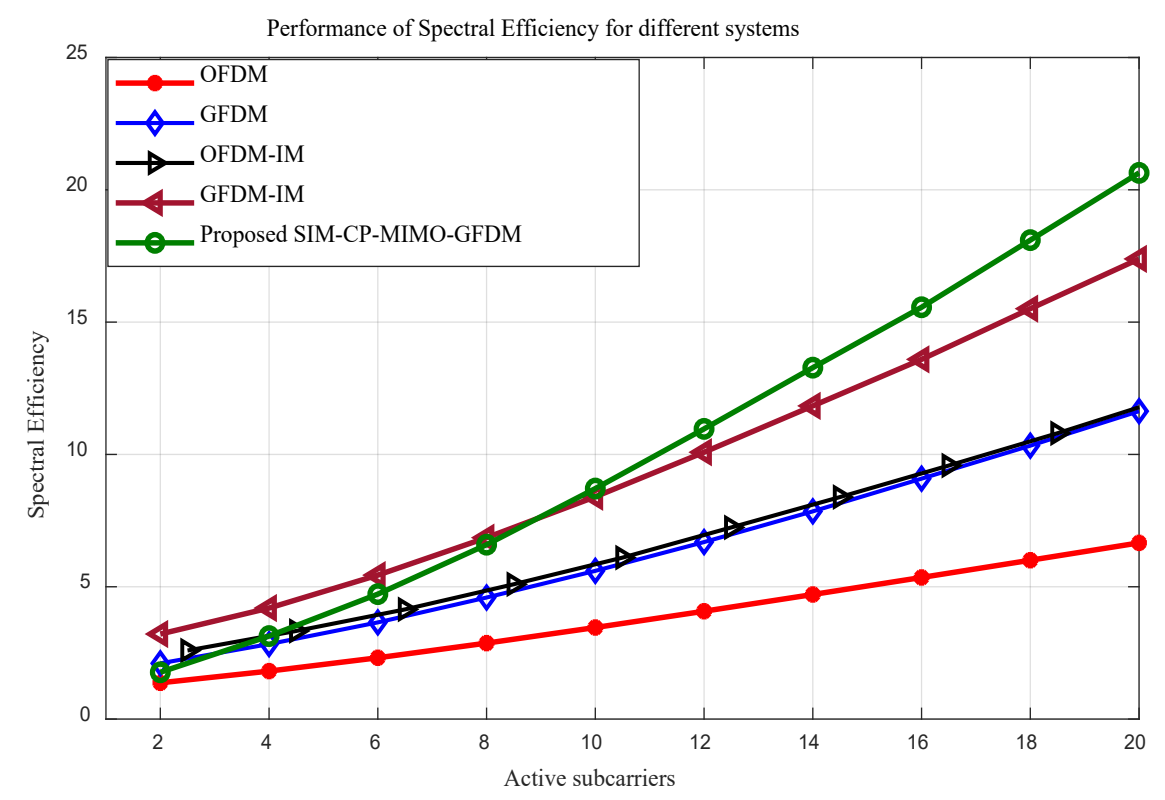

Figure 4: Performance of Spectral Effieciency for different systems

\section{Energy Efficiency}

It is defined two aspects. On device side it is quantity of information bits per unit of energy consumption of the communication module (in bit/Joule). Network side, the energy efficiency of a transmitter is defined the number of bits transmitted per unit joule energy (bits/Joule). According research article [31], the energy efficiency is depends on spectral efficiency as shown in equation ()

$$
\text { Energy Efficiency }(E E)=\frac{B_{T} S E}{P_{T}}=\frac{N \Delta f S E}{P_{T}} \text {. }
$$

Where, the term $B_{T}(=N \Delta f)$ is total bandwidth of the whole system. Where, $\Delta f$ is spacing between carrier signals and $\mathrm{N}$ is total number of subcarriers. The term $P_{T}$ is representing the total power consumption system which is including the transmit power $N P / \mathrm{n}$ and circuit power $P_{c}$. For simplification purpose, we are assumed $N P / \mathrm{n}>P_{c}$ because few subcarriers are activated based on index bits. Therefore, improved energy efficiency is calculated for a system as shown in equation ().

$$
\text { Energy Efficiency }(E E)=\frac{n \Delta f S E}{P} \quad P<P_{T}
$$


Figure 5 represent the performance analysis of energy efficiency versus throughput of the system. It is clear from the analysis that the energy efficiency of proposed scheme is gradually increased with increasing throughput of the system. Moreover, the energy efficiency is constant for higher values of throughput that means system maintain good performance. Where, proposed scheme that SIM-CP-MIMO-GFDM achieved 11.6 bits/Joules than compared to existing methods like OFDM, GFDM, OFDM-IM and GFDMIM. We are recommended proposed scheme to future wireless communications due to improvement in spectral and energy efficiency.

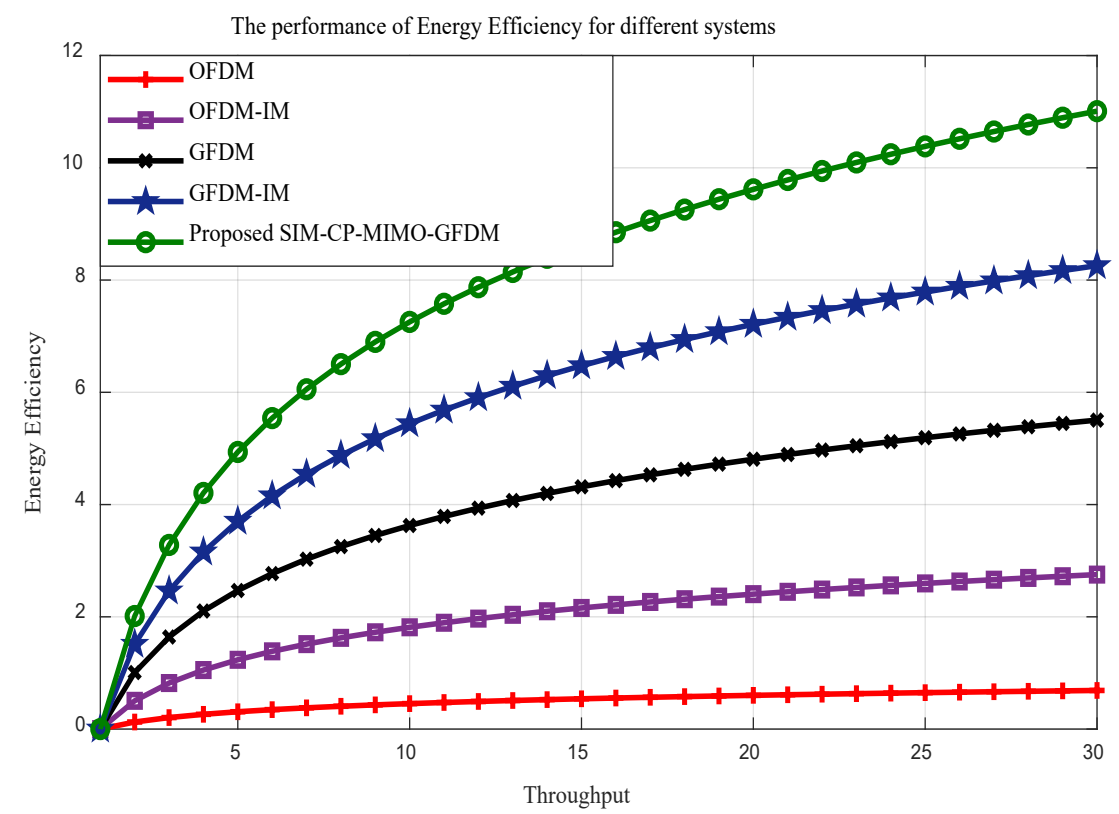

Figure 5: Performance of Energy Effieciency for different systems

\section{Channel Capacity}

All practical channels are noisy channels. There are no noiseless channels. According to statement of Shannon capacity theorem maximum acceptable data rate for a practical noise channels

Channel capacity $=B W \cdot \log _{2}(1+S N R)$

Where, channel capacity measured in bits per second and BW represent the Bandwidth. BW is a fixed quantity and we cannot modify. Here, SNR = signal power / noise power and channel capacity directly depends on Signal to Noise ratio. The advantages of MIMO antenna system in propose scheme such as beam forming, good diversity order help to increase the 
channel capacity even in bad channel conditions. Figure 6 shows that the proposed scheme achieved channel capacity up to 22.2 bit per second at $\mathrm{SNR}=20$ as compared to existing techniques.

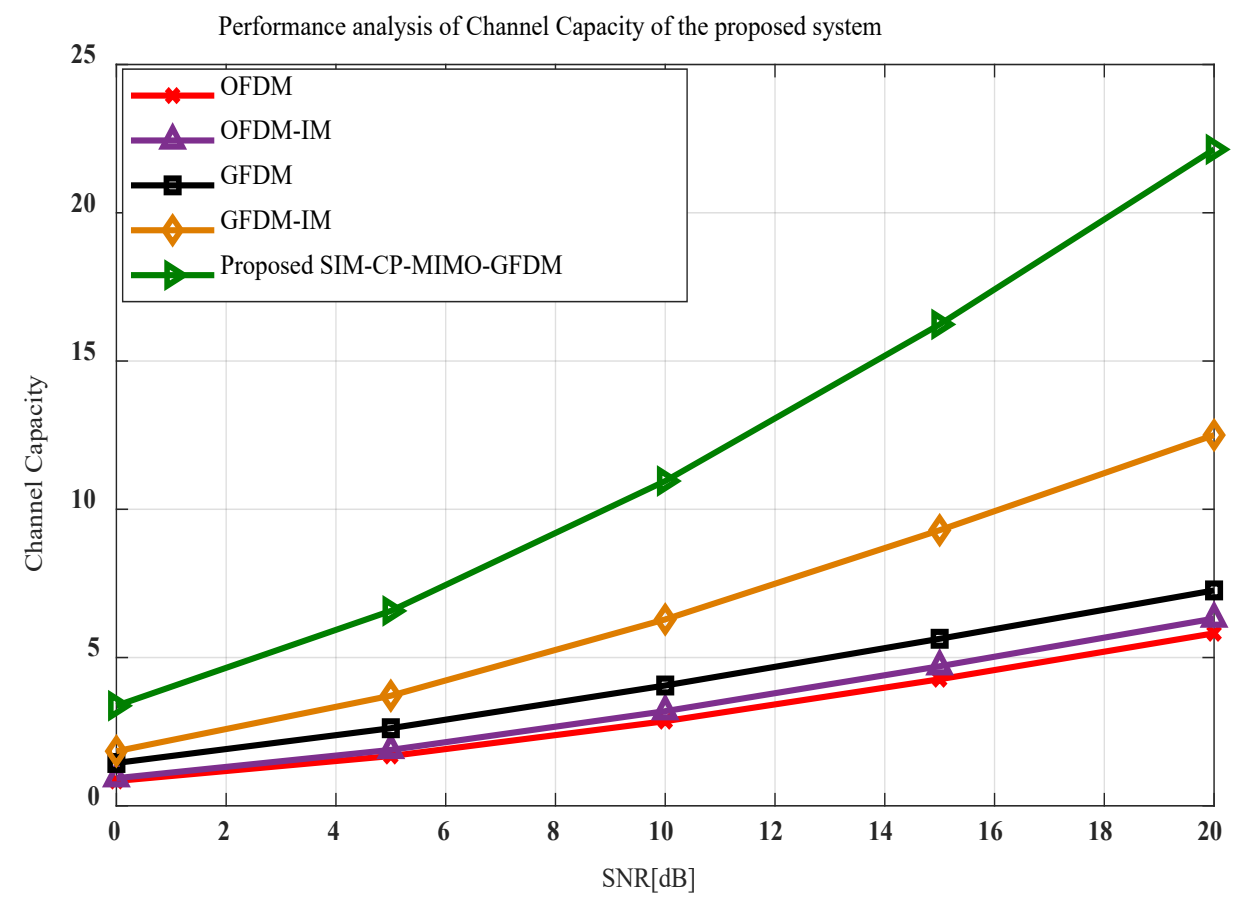

Figure 6: Performance of Channel Capacity for different systems

\section{Bit error rate}

Bit error rate is defined as which the ratio between the numbers of wrong bits is received by receiver to total transmitted bits. In proposed scheme In-phase and quadrature phase channels are independent. Hence, the performance of bit errors can be analysed separately. According to union bounding technique the BER of the proposed scheme upper bounded by following expression

$$
P_{e} \leq \frac{1}{p 2 p} \sum_{X_{\beta}} \sum_{\hat{X}_{\beta}} \operatorname{Pr}\left(X_{\beta}^{I} \rightarrow \hat{X}_{\beta}^{I}\right) \times \operatorname{Pr}\left(X_{\beta}^{Q} \rightarrow \hat{X}_{\beta}^{Q}\right) e\left(X_{\beta}, \hat{X}_{\beta}\right)
$$

Where, the term $e\left(X_{\beta}, \hat{X}_{\beta}\right)$ is representing the amount of bit variations from $X_{\beta}$ to $\hat{X}_{\beta}$ Figure 7 shows that the BER performance of proposed scheme with other existing methods in Raleigh fading channel using QAM constellation and with 4 transmitting antennas. In this scheme, in-phase and quadrature phase components are selected together. Therefore, it conveys extra bits per subcarrier group and encoded more active elements and also index bits 
spreading over subcarriers. We also achieved harvesting extra diversity gain with help constellation precoding. By using QR decomposition based ML detection process help to detect the subsequent SIM-GFDM blocks successfully. Figure 7 illustrates the proposed scheme bit error rate performance is improved. It is achieved BER value up to $1.66 \times 10^{-5}$ at $\mathrm{SNR}=10 \mathrm{~dB}$ is better than OFDM, GFDM, GFDM-IM, OFDM-IM methods.

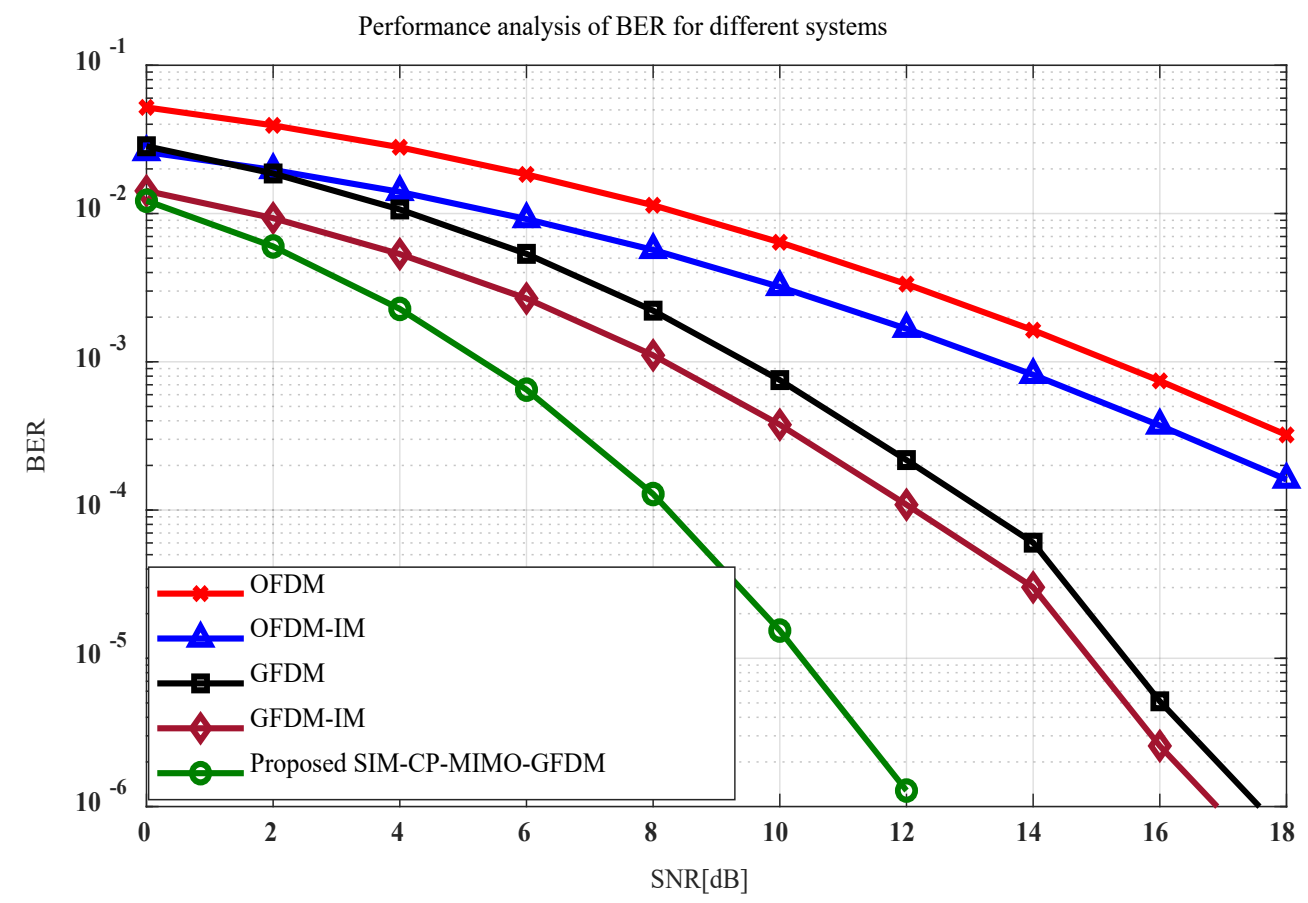

Figure 7: Performance of Bit error rate for different systems

Figure 8 represents a similar Bit error rate comparison with and without constellation precoding techniques. This is validating the effectiveness of constellation precoding for both SIM-OFDM and SIM-GFDM systems. In this scheme, constellation sysmbols are multiplied with optimal precoding matrix. The choices of an optimal rotation angles are effect the average bit error rate probability. These optimal rotation angles are computed through iterations in computer search. Therefore, generated pair of symbols rotated and it becomes diamond shaped $M^{2}-Q A M$ constellation. This increase the transmit diversity and coding gains but existing STC, TR-STC precoders increased the loss rate due to some amount of data used for the maintain full orthogonality also not possible to achieve the full diversity gain. In figure 8, SIM-CP-MIMO-GFDM and Precoded SIM-MIMO-OFDM schemes achieved good BER performance up to $10^{-5}$ and $1.86 \times 10^{-5}$ values at $\mathrm{SNR}=10 \mathrm{~dB}$ respectively than compared to without using precoding methods. 


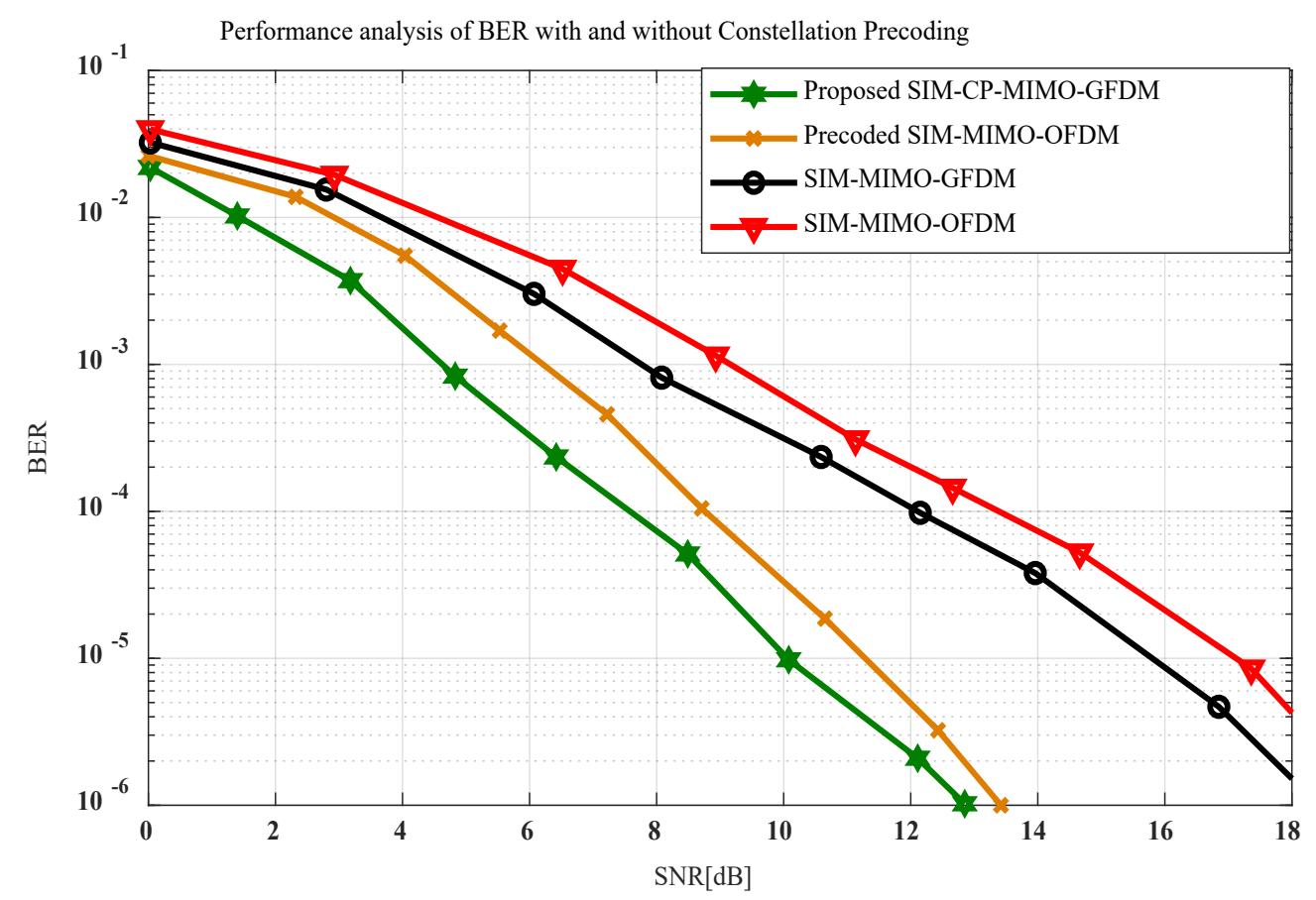

Figure 8: Performance of Bit error rate with and without precoding technique

In figure 9, the BER performance of different detection schemes has been compared with proposed QRD based ML detector. Here, proposed scheme is assumed in-phase and quadrature phase index modulation with $\left(n, k^{I}, k^{Q}\right)=(4,2,2)$ and QAM modulation. Transmitter and receiver designed with $4 \mathrm{x} 4 \mathrm{MIMO}$ antenna system configuration. From the figure we can understand that the MMSE, LLR and ML detection schemes provides the worst BER performance respectively as compared with proposed scheme. Maximum Likelihood scheme do not provide the good BER due to its non-orthogonality, individual subcarrier filtering and more computational complexity. And also, LLR and MMSE methods not provide the simultaneous detection of index modulation bits, MIMO and GFDM modulation bits in an efficient way but it is possible with SIM-CP-MIMO-GFDM scheme and also maintains the trade-off between computational complexity and BER performance. Finally, we can concluded proposed scheme achieves the BER value $10^{-6}$ at $\mathrm{SNR}=9 \mathrm{~dB}$. 


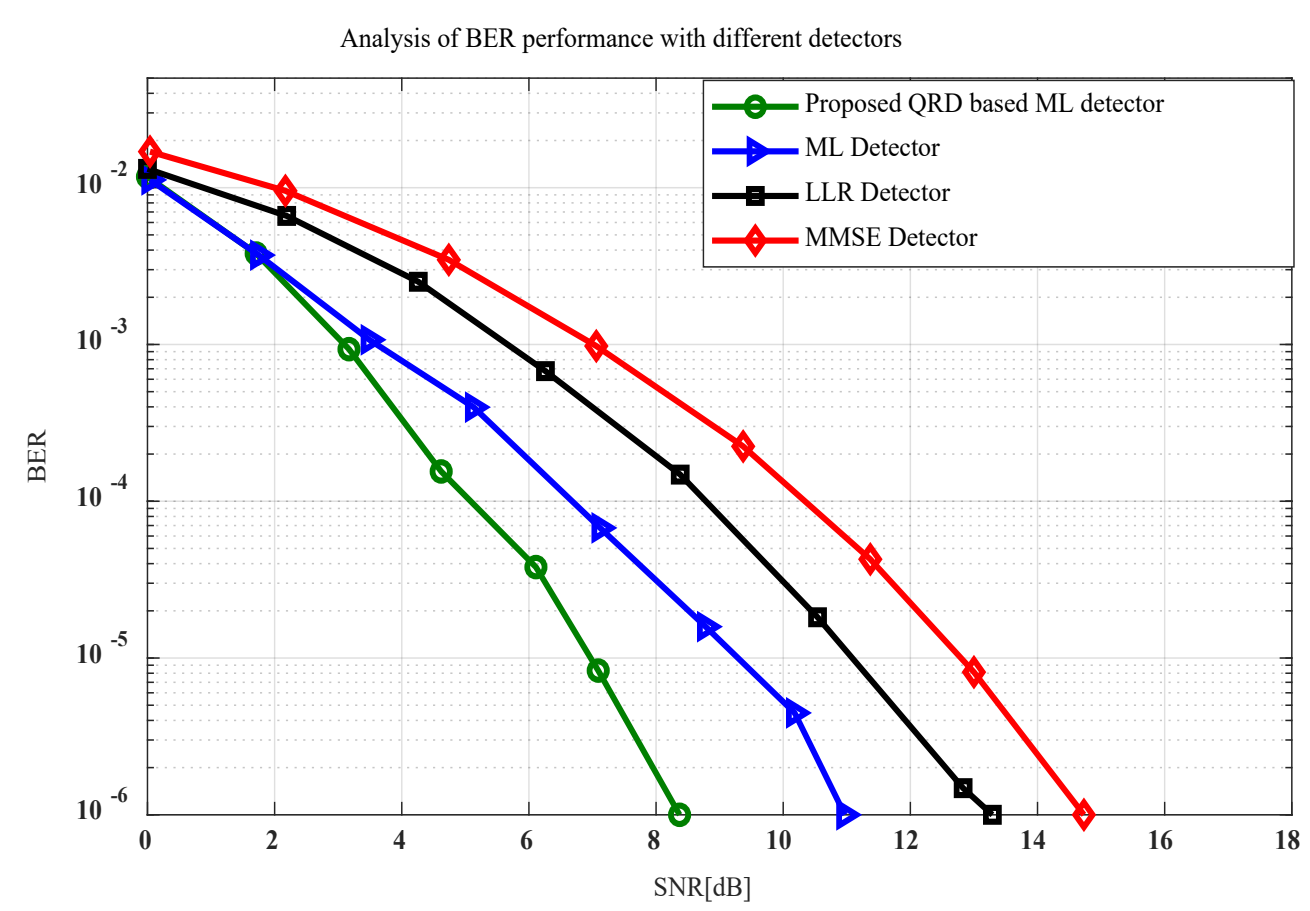

Figure 9: Performance of Bit error rate for different detecting technques

Table 3 represent the performance of Spectral Efficiency, Energy Efficiency, Channel Capacity and Bit error rate comparisons among OFDM, OFDM-IM, GFDM, GFDM-IM and SIM-CP-MIMO-GFDM systems. From the table, performance matrices values are gradually increased from conventional system to proposed scheme. Thus, the proposed scheme has suitable for future wireless communications due to enhanced data rates, reliability, and flexibility.

Table 3: Performance comparison of proposed scheme with existing methods

\begin{tabular}{|l|c|c|c|c|c|}
\hline \multicolumn{1}{|c|}{ Parameter } & OFDM & OFDM-IM & GFDM & GFDM-IM & Proposed scheme \\
\hline $\begin{array}{l}\text { Spectral Efficiency@ } \\
\text { active subcarriers 20 }\end{array}$ & 7.1 & 12.1 & 12.2 & 17.5 & 20.8 \\
\hline $\begin{array}{l}\text { Energy Efficiency@ } \\
\text { throughput=30Mbps }\end{array}$ & 0.8 & 2.8 & 3.7 & 8.1 & 11.6 \\
\hline $\begin{array}{l}\text { Channel Capacity@ } \\
\text { SNR=20dB }\end{array}$ & 5.8 & 6.2 & 7.1 & 12.5 & 22.2 \\
\hline $\begin{array}{l}\text { Bit error } \\
\text { rate@SNR=12dB }\end{array}$ & $6.35 \times 10^{-3}$ & $3.17 \times 10^{-3}$ & $7.78 \times 10^{-4}$ & $3.89 \times 10^{-4}$ & $1.66 \times 10^{-5}$ \\
\hline
\end{tabular}




\section{CONCLUSION}

Index Modulation plays an important role in present transmission methods by combining with Multicarrier modulation methods such as OFDM, GFDM and MIMO antenna systems. But, the research is going on GFDM-IM system to enhance the spectral efficiency and to achieve the better diversity order. In this research article, we combined the index modulation with GFDM, MIMO antenna transmission system and constellation precoding to efficient transmission of data for future wireless communication systems. The spectral efficiency of the proposed technique was improved by activated subcarriers in innovative way on both In-phase and Quadrature phase branches. Additionally, we achieved the diversity gain by rotating constellation symbols by using optimal constellation precoding. Therefore, bit error rate performance of SIM-CP-MIMO-GFDM is improved up to $1.66 \times 10^{-5}$ with constellation precoding. GFDM is a block based multicarrier modulation scheme. Therefore, at the receiver side QR-decomposition based ML detector is used to detect the GFDM blocks with minimum errors as compared to existing methods. The proposed scheme achieved $20.8 \mathrm{bits} / \mathrm{sec} / \mathrm{Hz}$ spectral efficiency at 20 active subcarriers, 11.6 bits $/ \mathrm{joule} / \mathrm{Hz}$ energy efficiency at $30 \mathrm{Mbps}$ throughput and $22.2 \mathrm{bps}$ channel capacity at $20 \mathrm{~dB}$ SNR when compared to conventional OFDM, GFDM, and OFDM-IM and GFDM-IM schemes.

\section{Acknowledgements:}

Part of this work this work supported by Aditya College of Engineering Technology and R \& D Laboratory in JNTUK University, Kakinada, Andhra Pradesh, India.

\section{References :}

[1] M. Polese, R. Jana, V. Kounev, K. Zhang, S. Deb, and M. Zorzi, "Machine learning at the edge: A data-driven architecture with applications to $5 \mathrm{G}$ cellular networks," IEEE Trans. Mob. Comput., 2020.

[2] J. G. Andrews et al., "What Will 5G Be?," IEEE J. Sel. Areas Commun., vol. 32, no. 6, pp. 1065-1082, 2014, doi: 10.1109/JSAC.2014.2328098.

[3] A. Gupta and R. K. Jha, "A Survey of 5G Network: Architecture and Emerging Technologies," IEEE Access, vol. 3, pp. 1206-1232, 2015, doi: 10.1109/ACCESS.2015.2461602.

[4] M. Agiwal, A. Roy, and N. Saxena, "Next Generation 5G Wireless Networks: A Comprehensive Survey," IEEE Commun. Surv. Tutorials, vol. 18, no. 3, pp. 16171655, 2016, doi: 10.1109/COMST.2016.2532458.

[5] T. E. Bogale and L. B. Le, "Massive MIMO and mmWave for 5G Wireless HetNet: Potential Benefits and Challenges," IEEE Veh. Technol. Mag., vol. 11, no. 1, pp. 64 75, 2016, doi: 10.1109/MVT.2015.2496240.

[6] P. Angeletti and R. De Gaudenzi, “A Pragmatic Approach to Massive MIMO for 
Broadband Communication Satellites," IEEE Access, vol. 8, pp. 132212-132236, 2020, doi: 10.1109/ACCESS.2020.3009850.

[7] S.-M. Tseng, Y.-F. Chen, and H.-H. Fang, "Cross PHY/APP Layer User Grouping and Power Allocation for Uplink Multiantenna NOMA Video Communication Systems," IEEE Syst. J., vol. 14, no. 3, pp. 3351-3359, 2020, doi: 10.1109/JSYST.2019.2943601.

[8] S.-M. Tseng, C.-S. Tsai, and C.-Y. Yu, "Outage-Capacity-Based Cross Layer Resource Management for Downlink NOMA-OFDMA Video Communications: NonDeep Learning and Deep Learning Approaches," IEEE Access, vol. 8, pp. 140097140107, 2020, doi: 10.1109/ACCESS.2020.3004865.

[9] V. Petrov et al., "Dynamic Multi-Connectivity Performance in Ultra-Dense Urban mmWave Deployments," IEEE J. Sel. Areas Commun., vol. 35, no. 9, pp. 2038-2055, 2017, doi: 10.1109/JSAC.2017.2720482.

[10] N. Chen, S. Sun, M. Kadoch, and B. Rong, "SDN Controlled mmWave Massive MIMO Hybrid Precoding for 5G Heterogeneous Mobile Systems," Mob. Inf. Syst., vol. 2016, pp. 1-10, Mar. 2016, doi: 10.1155/2016/9767065.

[11] J. Mrkic, E. Kocan, and M. Pejanovic-Djurisic, "Index modulation techniques in OFDM relay systems for 5G wireless networks," in 2017 40th International Conference on Telecommunications and Signal Processing (TSP), 2017, pp. 208-211, doi: 10.1109/TSP.2017.8075970.

[12] M. Barbuto, A. Alù, F. Bilotti, and A. Toscano, "Exploiting Vortex Modes in the Design of Patch Antennas for Pattern Diversity and MIMO Systems," in 2020 Fourteenth International Congress on Artificial Materials for Novel Wave Phenomena (Metamaterials), 2020, pp. 93-95, doi: 10.1109/Metamaterials49557.2020.9284992.

[13] E. Yoon, S. Kim, S. Kwon, and U. Yun, “An Efficient Index Mapping Algorithm for OFDM-Index Modulation,” IEEE Access, vol. 7, pp. 184194-184206, 2019, doi: 10.1109/ACCESS.2019.2960572.

[14] E. Öztürk, E. Basar, and H. A. Çirpan, "Generalized Frequency Division Multiplexing With Flexible Index Modulation,” IEEE Access, vol. 5, pp. 24727-24746, 2017.

[15] S. Kalia, A. Joshi, and A. Agrawal, "PAPR Analysis of IFFT and DWT Based OFDMIM System," in 2019 International Conference on Signal Processing and Communication (ICSC), 2019, pp. 109-112, doi: 10.1109/ICSC45622.2019.8938217.

[16] E. Bașar, "Spatial modulation techniques for 5G wireless networks," in 2016 24th Signal Processing and Communication Application Conference (SIU), 2016, pp. 777780, doi: 10.1109/SIU.2016.7495855.

[17] A. M. Abu-Hudrouss, M.-. O. E. Astal, A. H. Al Habbash, and S. Aïssa, "Signed Quadrature Spatial Modulation for MIMO Systems," IEEE Trans. Veh. Technol., vol. 69, no. 3, pp. 2740-2746, 2020, doi: 10.1109/TVT.2020.2964118.

[18] B. Zheng, F. Chen, M. Wen, F. Ji, H. Yu, and Y. Liu, "Low-Complexity ML Detector and Performance Analysis for OFDM With In-Phase/Quadrature Index Modulation," IEEE Commun. Lett., vol. 19, no. 11, pp. 1893-1896, 2015, doi: 10.1109/LCOMM.2015.2474863.

[19] J. Li, M. Wen, X. Cheng, Y. Yan, S. Song, and M. Lee, "Generalised Pre-coding Aided Quadrature Spatial Modulation," IEEE Trans. Veh. Technol., vol. 66, p. 1, Jan. 2016, doi: 10.1109/TVT.2016.2565618.

[20] M. Wen, Q. Li, E. Basar, and W. Zhang, "Generalized Multiple-Mode OFDM With Index Modulation," IEEE Trans. Wirel. Commun., vol. 17, no. 10, pp. 6531-6543, 2018, doi: 10.1109/TWC.2018.2860954.

[21] E. Başar, "Multiple-Input Multiple-Output OFDM with Index Modulation," IEEE Signal Process. Lett., vol. 22, no. 12, pp. 2259-2263, 2015, doi: 10.1109/LSP.2015.2475361. 
[22] Y. Xiao, S. Wang, L. Dan, X. Lei, P. Yang, and W. Xiang, "OFDM With Interleaved Subcarrier-Index Modulation," IEEE Commun. Lett., vol. 18, no. 8, pp. 1447-1450, 2014, doi: 10.1109/LCOMM.2014.2332340.

[23] R. A. Kumar and K. S. Prasad, "Out-of-Band Radiation, PAPR and SER Analysis for Future Wireless (5G) Communications," 2018.

[24] N. Michailow et al., "Generalized Frequency Division Multiplexing for 5th Generation Cellular Networks," IEEE Trans. Commun., vol. 62, no. 9, pp. 3045-3061, 2014.

[25] R ANIL KUMAR and Dr.Ksatya Prasad, "JNTUK national conference," 2015.

[26] E. Öztürk, E. Basar, and H. A. Çırpan, "Generalized Frequency Division Multiplexing With Flexible Index Modulation Numerology," IEEE Signal Process. Lett., vol. 25, no. 10, pp. 1480-1484, 2018.

[27] M. Matthé, L. L. Mendes, N. Michailow, D. Zhang, and G. Fettweis, "Widely Linear Estimation for Space-Time-Coded GFDM in Low-Latency Applications," IEEE Trans. Commun., vol. 63, no. 11, pp. 4501-4509, 2015.

[28] B. T. Vo, H. H. Nguyen, and N. Quoc-Tuan, "Spatial modulation for OFDM with linear constellation precoding," in 2015 International Conference on Advanced Technologies for Communications (ATC), 2015, pp. 226-230, doi: 10.1109/ATC.2015.7388324.

[29] J. Li, Q. Li, S. Dang, M. Wen, X. Jiang, and Y. Peng, "Low-Complexity Detection for Index Modulation Multiple Access," IEEE Wirel. Commun. Lett., vol. 9, no. 7, pp. 943-947, 2020, doi: 10.1109/LWC.2020.2974730.

[30] W. D. Dias, L. L. Mendes, and J. J. P. C. Rodrigues, "Low Complexity GFDM Receiver for Frequency-Selective Channels," IEEE Commun. Lett., vol. 23, no. 7, pp. 1166-1169, 2019.

[31] Z. Hu, S. Lin, B. Zheng, F. Chen, Q. Wang, and Y. Wei, "Low-Complexity SubcarrierWise Detection for MIMO-OFDM with Index Modulation," IEEE Access, vol. PP, p. 1, Aug. 2017, doi: 10.1109/ACCESS.2017.2743744.

[32] R. A. Kumar and K. S. Prasad, "Performance analysis of an efficient linear constellation precoded generalized frequency division multiplexing with index modulation in 5G heterogeneous wireless network," Int. J. Commun. Syst., p. e4732.

[33] M. Wen, B. Ye, E. Basar, Q. Li, and F. Ji, "Enhanced Orthogonal Frequency Division Multiplexing With Index Modulation," IEEE Trans. Wirel. Commun., vol. 16, no. 7, pp. 4786-4801, 2017, doi: 10.1109/TWC.2017.2702618.

[34] R. A. Kumar and K. S. Prasad, "Performance Analysis of GFDM Modulation in Heterogeneous Network for 5G NR," Wirel. Pers. Commun., 2020, doi: 10.1007/s11277-020-07791-4.

[35] T. Lyche, Numerical Linear Algebra and Matrix Factorizations. 2020. 


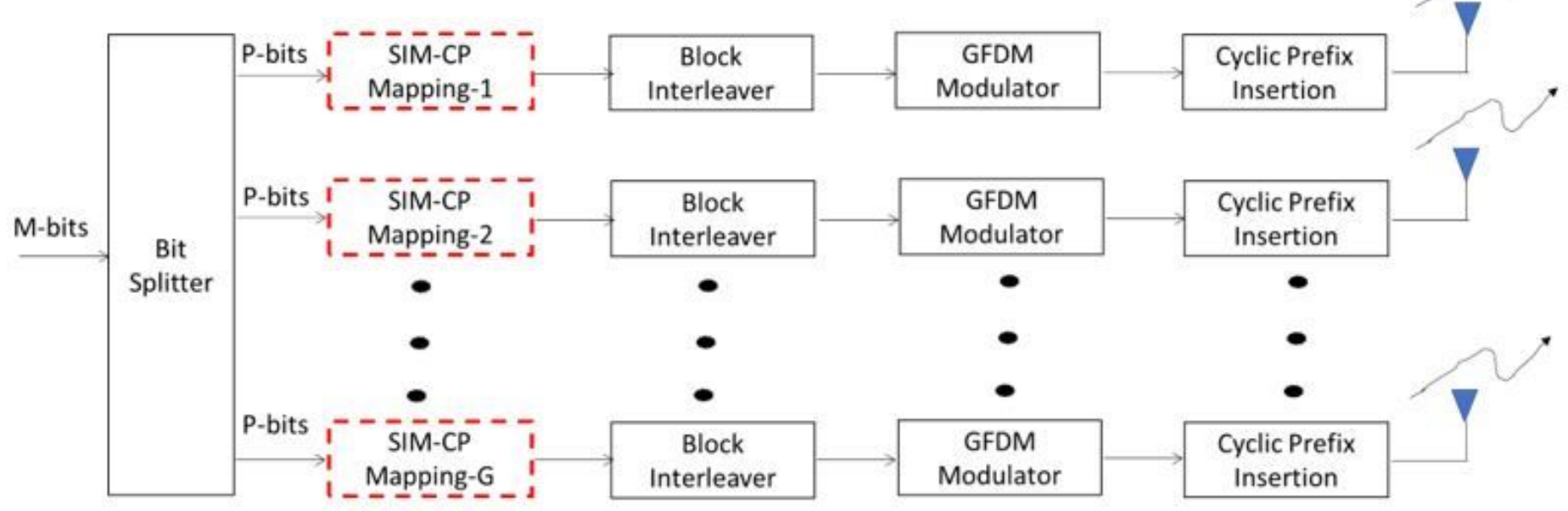

Figure 1

Proposed SIM-CP-MIMO-GFDM transmitter section

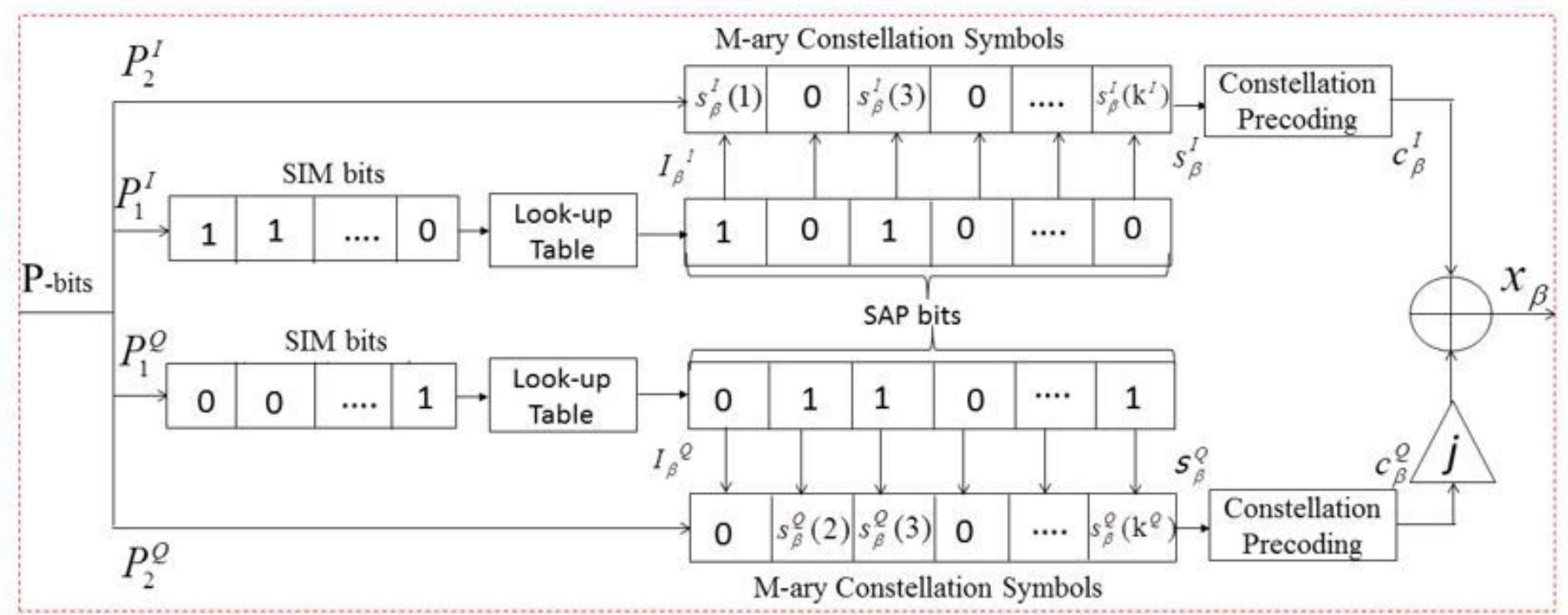

Figure 2

Block diagram of IM-CP Mapper 


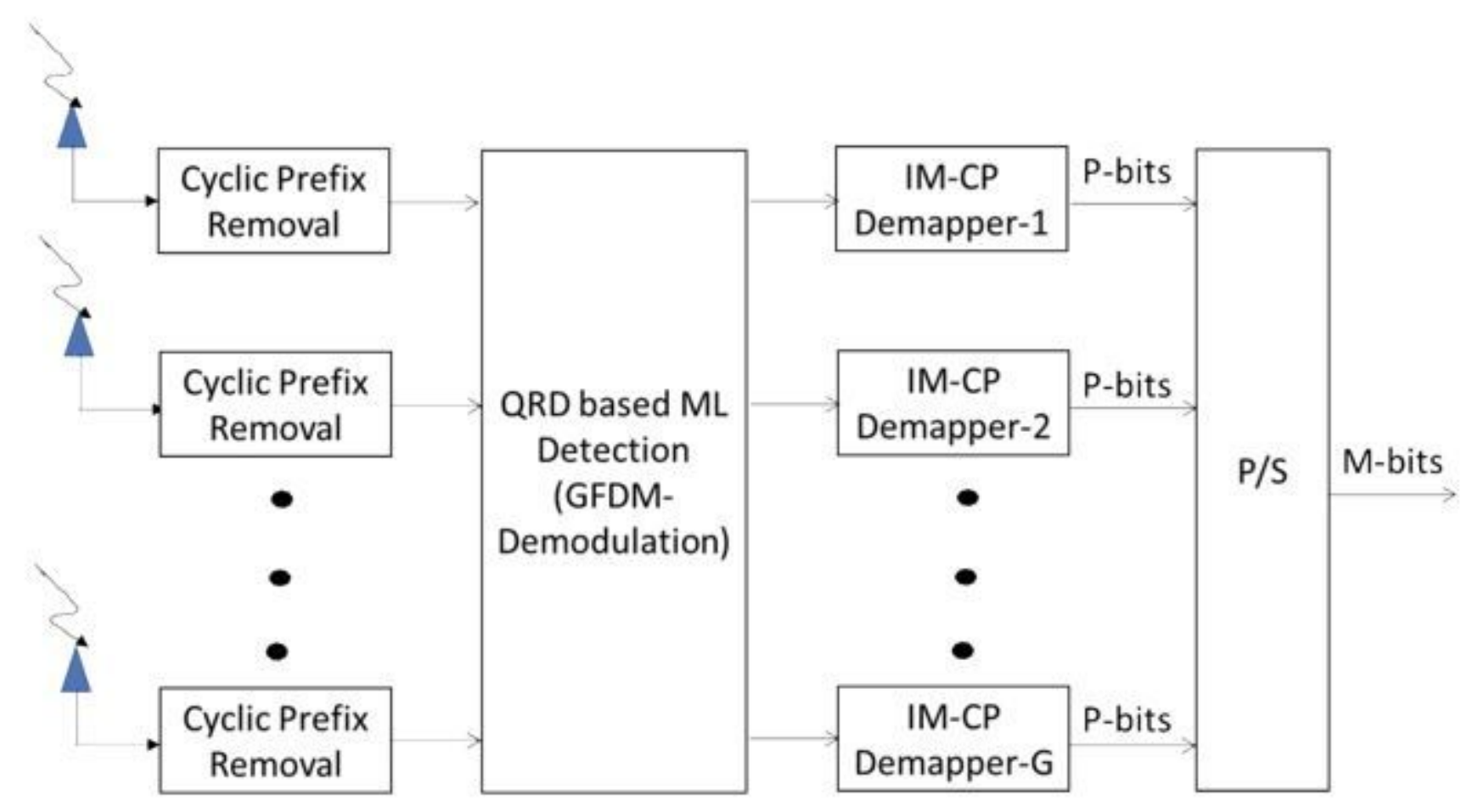

Figure 3

SIM-LP-MIMO-GFDM receiver with QR decomposition based ML detection

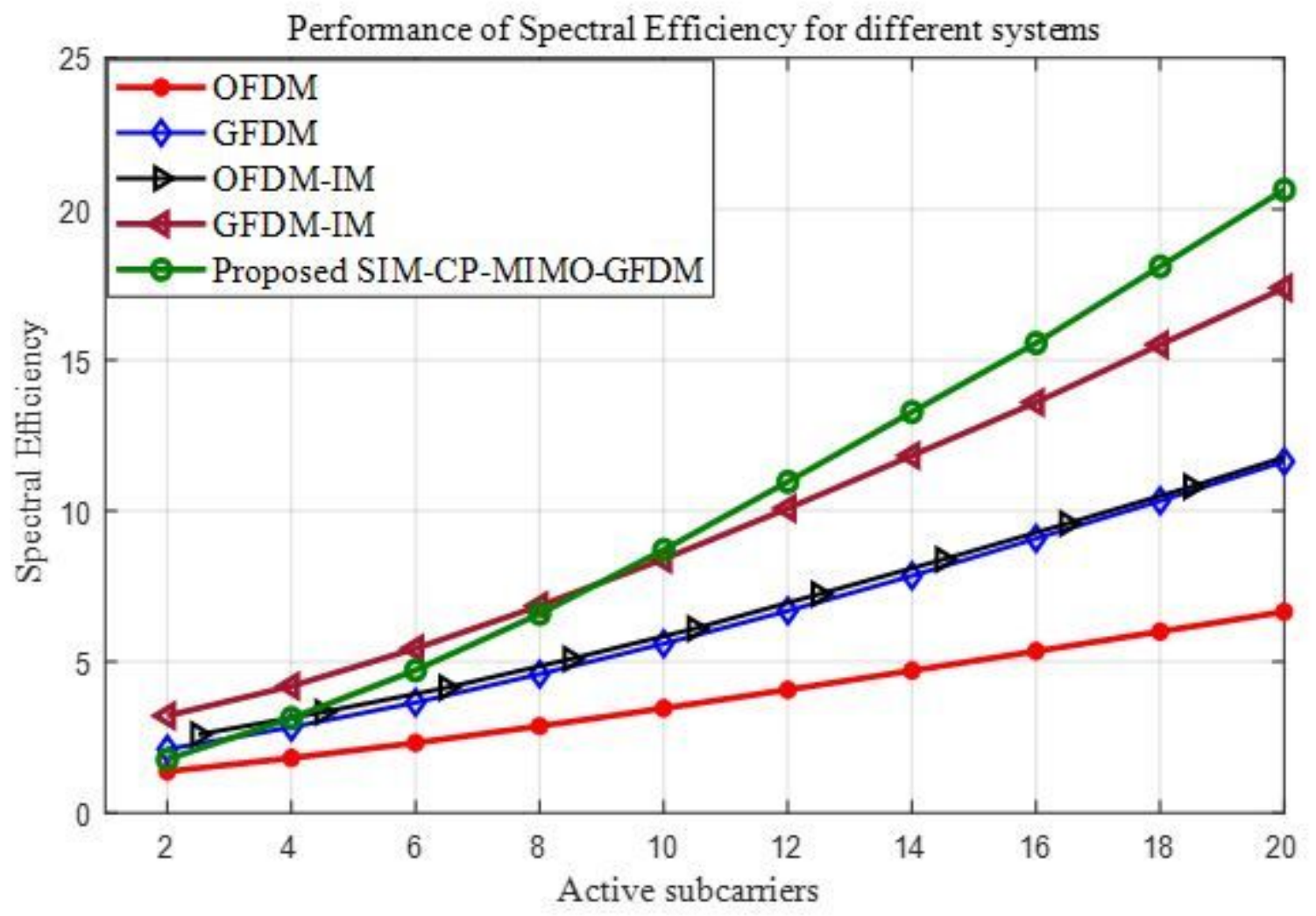

Figure 4 
Performance of Spectral Effieciency for different systems

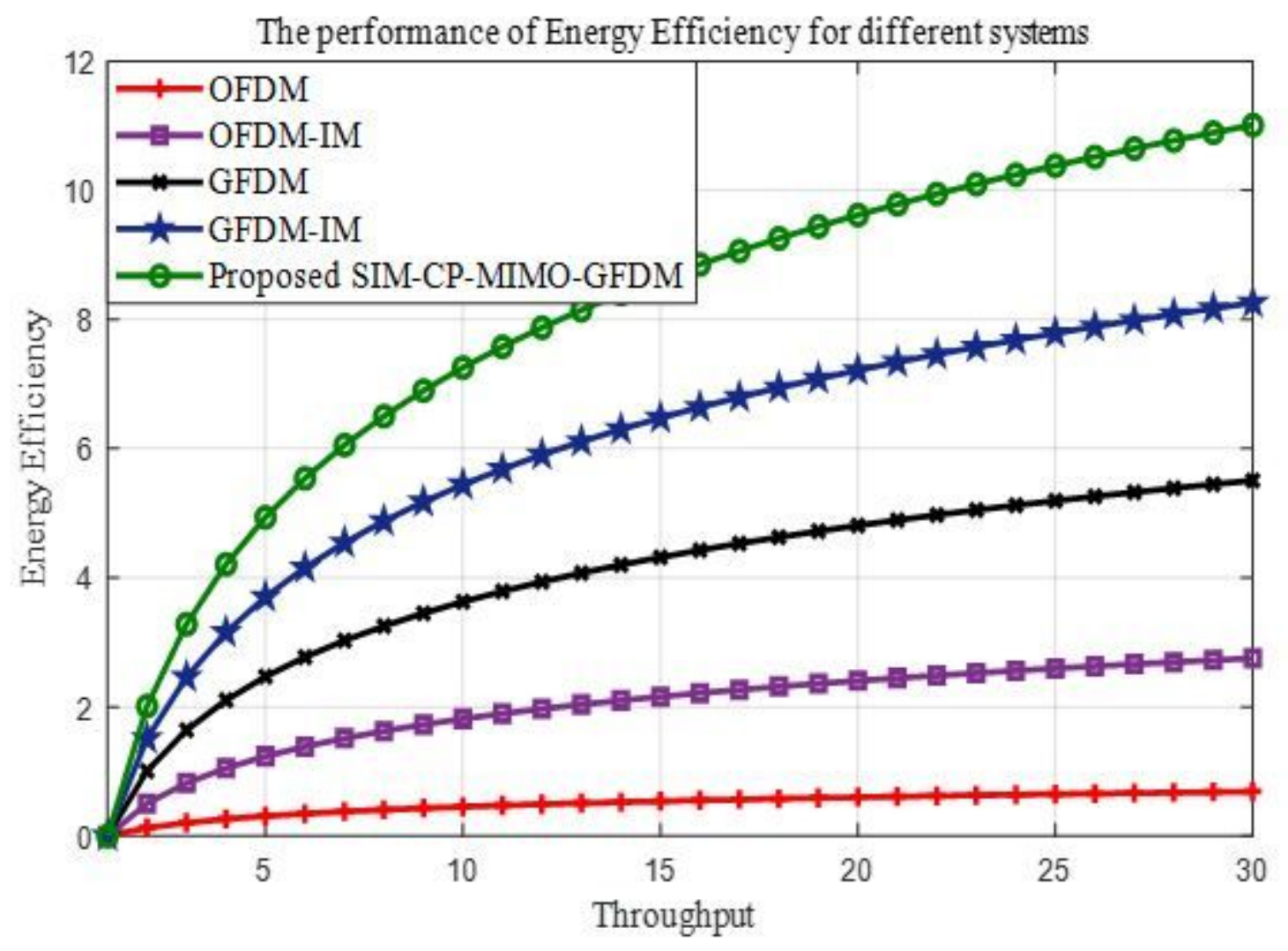

Figure 5

Performance of Energy Effieciency for different systems 


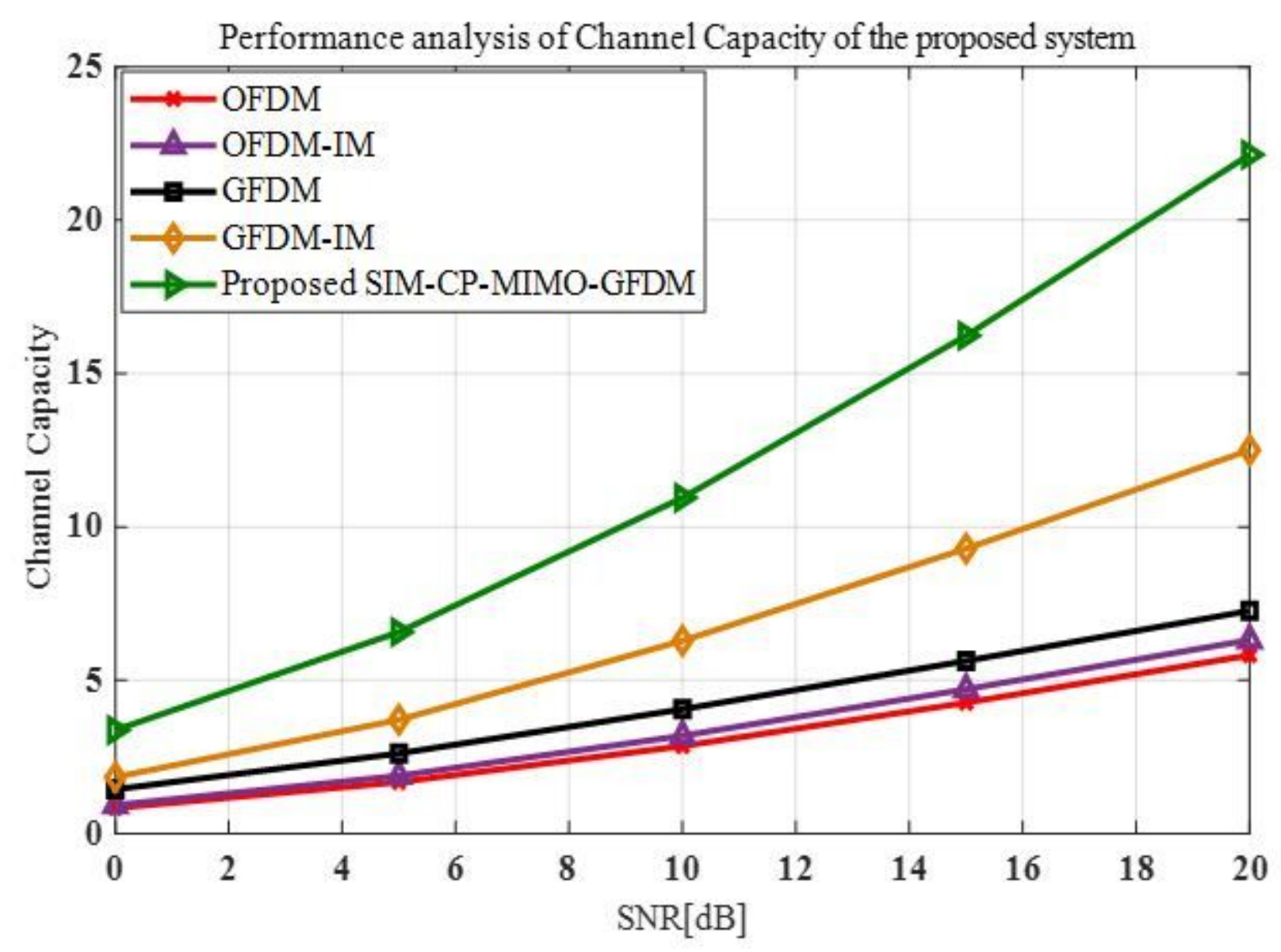

Figure 6

Performance of Channel Capacity for different systems 


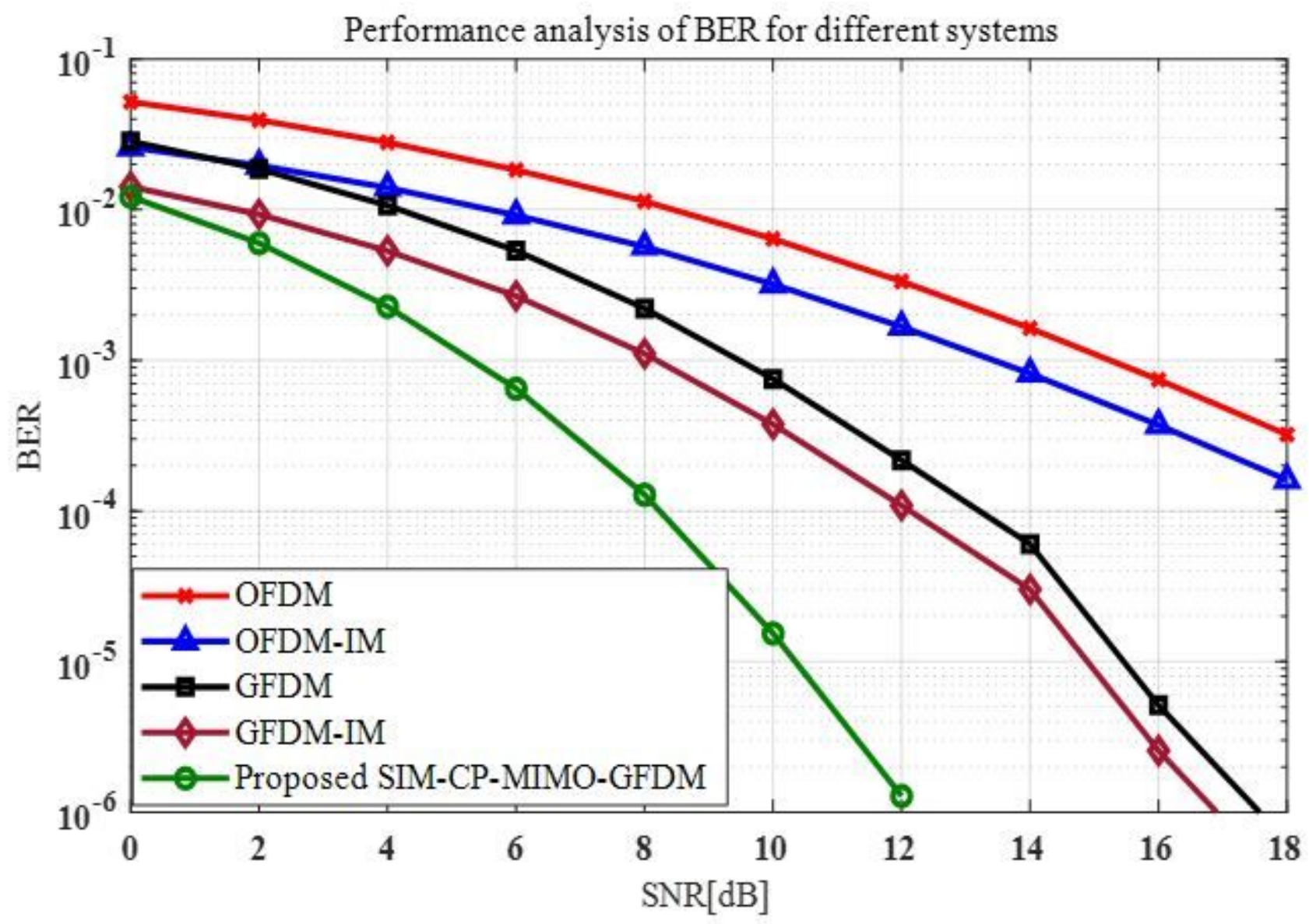

Figure 7

Performance of Bit error rate for different systems 


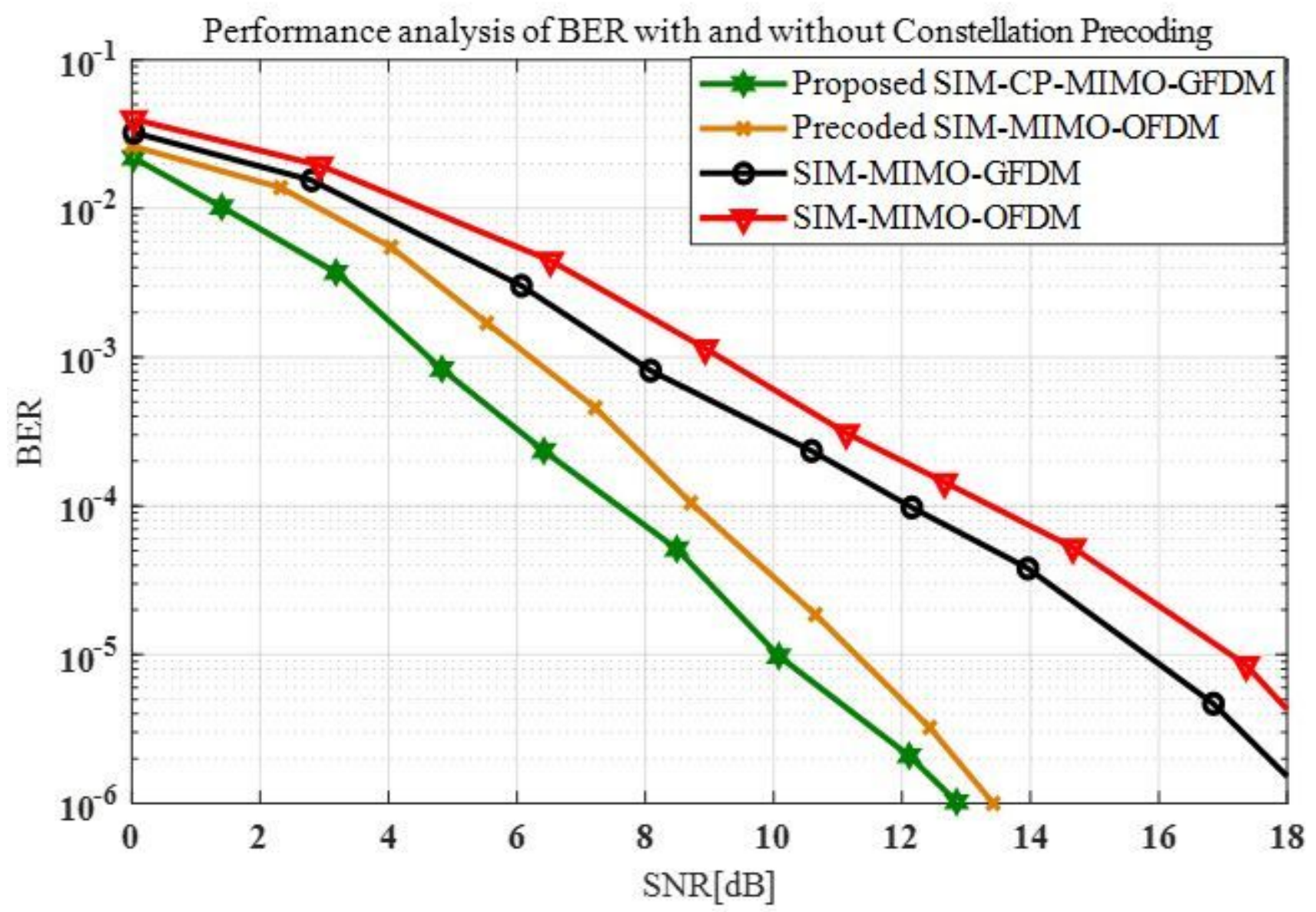

Figure 8

Performance of Bit error rate with and without precoding technique 


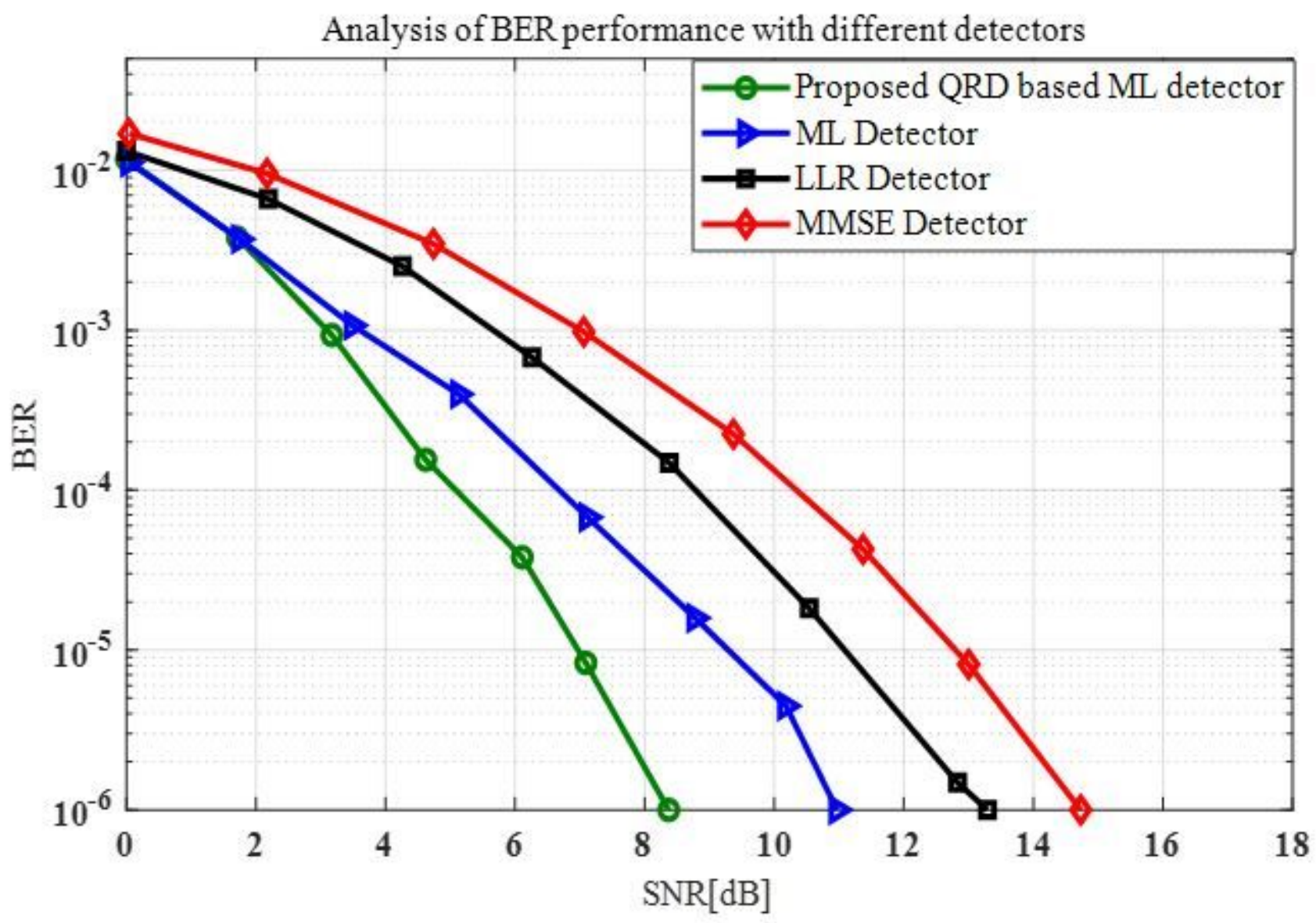

Figure 9

Performance of Bit error rate for different detecting technques 